\title{
Inter-Laboratory Uranium Double-Spike Experiment
}

\author{
G.P. Russ III
}

November 11, 1999

U.S. Department of Energy

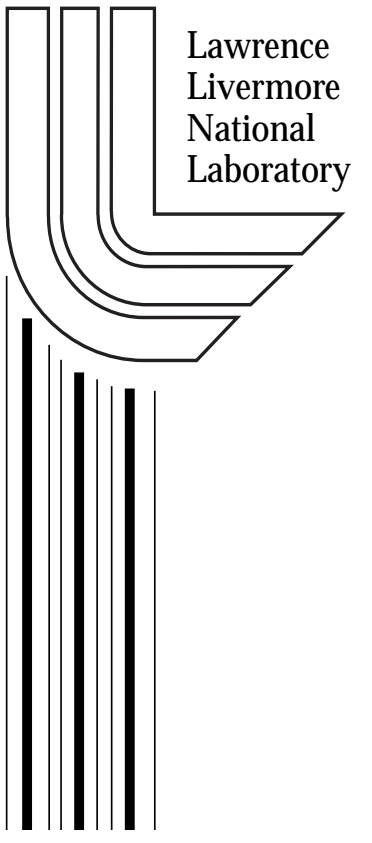




\section{DISCLAIMER}

This document was prepared as an account of work sponsored by an agency of the United States Government. Neither the United States Government nor the University of California nor any of their employees, makes any warranty, express or implied, or assumes any legal liability or responsibility for the accuracy, completeness, or usefulness of any information, apparatus, product, or process disclosed, or represents that its use would not infringe privately owned rights. Reference herein to any specific commercial product, process, or service by trade name, trademark, manufacturer, or otherwise, does not necessarily constitute or imply its endorsement, recommendation, or favoring by the United States Government or the University of California. The views and opinions of authors expressed herein do not necessarily state or reflect those of the United States Government or the University of California, and shall not be used for advertising or product endorsement purposes.

Work performed under the auspices of the U. S. Department of Energy by the University of California Lawrence Livermore National Laboratory under Contract W-7405-Eng-48.

This report has been reproduced directly from the best available copy.

Available to DOE and DOE contractors from the

Office of Scientific and Technical Information

P.O. Box 62, Oak Ridge, TN 37831

Prices available from (423) 576-8401

http://apollo.osti.gov/bridge/

Available to the public from the National Technical Information Service

U.S. Department of Commerce 5285 Port Royal Rd., Springfield, VA 22161 http://www.ntis.gov/

OR Lawrence Livermore National Laboratory Technical Information Department's Digital Library http://www.llnl.gov/tid/Library.html 


\title{
INTER-LABORATORY \\ URANIUM DOUBLE-SPIKE
}

EXPERIMENT

\author{
G. PRICE RUSS III
}

NOVEMBER 11, 1999 


\section{CONTRIBUTORS TO STUDY}

A. L. Boni, Savannah River Technology Center

D. W. Efurd, Los Alamos National Laboratory

D. Gerlach, Pacific Northwest National Laboratory

D. Hayes, Savannah River Technology Center

D. M. Hembree, Oak Ridge National Laboratory

R. Holdren, Pacific Northwest National Laboratory

D. Koppenaal, Pacific Northwest National Laboratory

G. Price Russ, Lawrence Livermore National Laboratory

C. Schick, Savannah River Technology Center

D. H. Smith, Oak Ridge National Laboratory 


\title{
INTER-LABORATORY URANIUM DOUBLE-SPIKE EXPERIMENT
}

\begin{abstract}
In environmental samples, the major analytical limitation on the use of uranium ${ }^{238} U /{ }^{235} U$ determinations as an indicator of uranium enrichment is mass dependent bias occurring during the measurement. The double-spike technique can be used to correct the data for this effect. The purpose of this experiment was to evaluate the variation of mass bias among several laboratories and to determine the extent to which the double-spike could be used to reduce analytical uncertainty. Four laboratories performed replicate analyses on each of three samples. Generally mass bias was determined to be small compared to the random scatter of the measurements, but in at least one case, the bias was $>1 \%$. In 8 of 12 cases, intra-laboratory variance was reduced when the double-spike correction was applied. For all three samples, the inter-laboratory variance was decreased, though the decrease was small. Based on a reasonable assumption about the "true" isotopic compositions of the samples, the accuracy of 11 of the twelve analyses was improved by applying the double spike correction. When the double spike is used to correct for mass bias, the ${ }^{238} \mathrm{U} /{ }^{235} \mathrm{U}$ accuracy is better than $1 \%$ even for samples as small as $1 \mathrm{ng}$. For 50 ng of uranium, $0.1 \%$ accuracy was achieved.
\end{abstract}

\section{Introduction}

The International Atomic Energy Agency (IAEA) operates a variety of programs designed to detect and/or monitor uranium isotopic enrichment efforts worldwide. The most direct indicator of such activity is the isotopic ratio ${ }^{238} \mathrm{U} /{ }^{235} \mathrm{U}$. The effectiveness of monitoring programs based on ${ }^{238} \mathrm{U} /{ }^{235} \mathrm{U}$ determinations depends on the precision and accuracy with which the determinations are made. This is particularly true for environmental samples where enriched effluent will be diluted by natural uranium in the environment.

The limiting uncertainty on the analytical results is the inability to correct for mass bias during the analysis. This bias results from a combination of mass-dependent transmission differences within mass spectrometers and in the case of thermal ionization mass spectrometry, TIMS, from mass-dependent volatility differences among the isotopes. (Lower mass isotopes tend to volatilize preferentially. This process is usually referred to as mass fractionation.)

For elements with two isotopes that occur in a constant ratio, the ratio of the measured ratio of those isotopes to the accepted value can be used to determine a mass bias correction factor. This factor can be used to correct the measured ratios of other isotopic pairs for mass bias and then look for non-mass-dependent variations in isotopic composition. The most commonly used mass bias correction is of the form

$$
\mathrm{R}_{\mathrm{t}}=\mathrm{R}_{\mathrm{m}}(1+\mathrm{n} \alpha)
$$

where $R_{t}$ and $R_{m}$ are the true and measured ratios, respectively, $n$ is the mass difference between the two isotopes being ratioed, and $\alpha$ is the mass bias per mass unit. 
In the case of uranium where enrichment is possible, there is no pair of isotopes that can be assumed to occur in a known ratio. For this reason, the apparent precision of a single determination of ${ }^{238} \mathrm{U} /{ }^{235} \mathrm{U}$ will be better than the analysis-to-analysis reproducibility. Even when one can control the analysis-to-analysis variation there is the possibility of systematic errors leading to inter-laboratory differences.

This difficulty can be overcome by the use of a "double spike". The double spike consists of a mixture of two isotopes for which the isotopic ratio is well known and which are of low abundance in the samples to be analyzed. In the case of uranium the isotopes ${ }^{236} \mathrm{U}$ and ${ }^{233} \mathrm{U}$ are the preferred choices because neither occurs in nature and their mass difference is the same as for the ${ }^{238} \mathrm{U}-{ }^{235} \mathrm{U}$ pair of interest. The ratio of the measured ${ }^{236} \mathrm{U} /{ }^{233} \mathrm{U}$ to the known ratio determines the correction to be applied to the measured ${ }^{238} \mathrm{U} /{ }^{235} \mathrm{U}$. Of course samples with an enriched uranium component, are likely to contain ${ }^{236} \mathrm{U}$ and possibly ${ }^{233} \mathrm{U}$. It is therefore necessary to measure the isotopic composition of both "spiked" and "unspiked" aliquots of the sample. It is then possible to solve for the bias in each analysis and to obtain the bias corrected ${ }^{238} \mathrm{U} /{ }^{235} \mathrm{U}$. (The double-spike technique and equations are discussed more fully in Appendix A.) While not of immediate interest for this work, the ratio of spike to sample can be used to determine concentration.

\section{Purpose}

The purposes of this experiment were to determine the extent of inter-laboratory variation in uranium isotopic analyses and how effectively use of the double spike reduces these differences. It was agreed in advance that each laboratory would receive three samples of "simulated river water", which consisted of a uranium standard with near natural isotopic composition, dried in a tube and an aliquot of double spike. Each laboratory was to receive aliquots of the same samples and spike so results could be directly compared.

\section{Experimental}

Los Alamos National Laboratory, LANL, prepared the samples from standards supplied by the IAEA and distributed them to the participants. Each tube contained approximately $750 \mathrm{ng}$ of uranium. To make the results as comparable as possible, each laboratory was requested to use $50 \mathrm{ng}$ per filament loading and to load three spiked and two unspiked aliquots of each sample. (They were given the option of analyzing smaller samples if they wished.)

The double spike was supplied by Lawrence Livermore National Laboratory, LLNL, as a liquid in a teflon vial. Because LLNL has a limited amount of double spike, each laboratory received only enough double spike for the immediate experiment. This amounted to $20 \mathrm{ng}$ of ${ }^{233} \mathrm{U}$ and ${ }^{236} \mathrm{U}$ (each) per laboratory. This was sufficient to spike each sample to ${ }^{233} \mathrm{U} /{ }^{235} \mathrm{U}=4$. The isotopic composition of the spike was also supplied so that each laboratory could independently make the double spike corrections. The optimal spiking level will depend on the purity of the double spike; ie, how free it is of ${ }^{238} \mathrm{U}$ and ${ }^{235} \mathrm{U}$, and the availability of the spike. In general terms, as long as the amount of spike 
isotopes added is a few times the amount of ${ }^{235} \mathrm{U}$ present, the overall uncertainty will be controlled by the uncertainty on the ${ }^{235} \mathrm{U}$ measurements rather than the uncertainty on the measurements of the spike isotopes.

Each laboratory was requested to report both measured and fractionation corrected isotopic ratios for each loading of each sample along with the approximate number of nanograms loaded LLNL, Oak Ridge National Laboratory (ORNL), Pacific Northwest National Laboratory (PNNL), and Savannah River Site (SRS) provided analytical data.

During the course of the experiment, the IAEA obtained, and made available to the participants in this study, another double spike. This spike is available in much larger quantities than the LLNL spike. This spike was distributed to the participating laboratories by ORNL. One laboratory analyzed the samples with respect to both spikes. Both sets of data and a preliminary comparison of the two double-spikes will be presented.

The utility of a double spike is directly dependent on knowing its isotopic composition. It is important to know both the ${ }^{236} \mathrm{U} /{ }^{233} \mathrm{U}$ and the amounts of ${ }^{235} \mathrm{U}$ and ${ }^{238} \mathrm{U}$ relative to the major isotopes. If ${ }^{235} \mathrm{U}$ and ${ }^{238} \mathrm{U}$ are present at significant levels in the spike, the uncertainty of ${ }^{238} \mathrm{U}^{235} \mathrm{U}$ will increase and could cancel the advantage of the technique. ${ }^{234} \mathrm{U}$ is not a concern as long as it is minor. Even for disequilibrium studies, ${ }^{238} \mathrm{U} \rightarrow{ }^{234} \mathrm{U}$, ${ }^{234} \mathrm{U}$ in the spike is not important unless one is trying to avoid analyzing an unspiked sample.

The LLNL double spike was prepared by mixing high purity ${ }^{233} \mathrm{U}$, SRM995, with ${ }^{236} \mathrm{U}$ that had been processed twice through an isotope separator. The ${ }^{236} \mathrm{U} /{ }^{233} \mathrm{U}$ was determined mass spectrometrically using mixtures of the double spike and SRM U500, an internationally recognized standard with a well characterized ${ }^{238} \mathrm{U}^{235} \mathrm{U}$ near unity. While not "absolute" this approach bases the isotopic ratio calibration on a readily available primary standard. The use of SRM995 directly provided a concentration standard as well. The author does not know the method used to prepare the IAEA double. The analyses from Laboratory L, presented here, provide a preliminary check on its alleged isotopic composition. The isotopic compositions of the LLNL and IAEA double spikes are summarized in Table 1.

\section{Results}

In this report the names of the institutions associated with particular results have been omitted. The institutions have been labeled I, J, K, and L. (These letters were used, in part, to avoid confusion with the identifiers $\mathrm{A}-\mathrm{E}$ used for a previous performance evaluation study.) Appendices B-E contain slightly edited copies of the reports originally submitted by the laboratories.

The amount of sample used varied from $50 \mathrm{ng}$ per analysis by one laboratory, I, to $1 \mathrm{ng}$ per analysis used by two laboratories, and $\mathrm{K}$ and L. Laboratory J used $15 \mathrm{ng}$. Laboratory $\mathrm{L}$ also performed two experiments with the IAEA double spike. One was using $1 \mathrm{ng}$ of 
uranium per analysis. The second used $15 \mathrm{ng}$ per analysis. While the focus of the study was the ability to determine ${ }^{238} \mathrm{U} /{ }^{235} \mathrm{U}$, data were also reported for the minor isotopes. Minor isotope data will not be discussed but are included in the tables and appendices.

Table II is a summary of the "measured" $238 / 235$ data relevant to the experiments using the LLNL spike. IN this and the following tables, the ratios tabulated for each laboratory are the averages for the analyses performed by that laboratory. The uncertainties are in terms of the standard deviation of the distribution of values rather than standard deviation of the mean.

Table III presents the mass-bias corrected isotopic ratios for the experiments using the LLNL spike. The tabulated values are the individual laboratories best estimates of the isotopic ratios in the samples. Minor isotopes have been included for completeness. Only one laboratory, I, reported data for ${ }^{233} \mathrm{U}$ in the samples. No ${ }^{233} \mathrm{U}$ was observed.

If one knew the "true" ${ }^{238} \mathrm{U} /{ }^{235} \mathrm{U}$ in the samples, one could ratio all the observations to the true values and compare the data from the various samples on a common scale rather than having to discuss the samples separately. Based on the data obtained in this experiment, it seems likely that the three samples were in fact the same samples used in the previous performance evaluation exercise. These samples were IRMM isotopic standards for which the "true" ratios have been made known to the laboratories. For the following discussion, it will be assumed that the samples are the IRMM standards and all data will be normalized to the standard with ${ }^{238} \mathrm{U} /{ }^{235} \mathrm{U}$ closest to the measured value. These assumed isotopic compositions are shown in Table IV. Even if the assumption is wrong, it provides convenient values for normalization. Statements concerning accuracy, however, depend directly on this assumption.

The normalized values for all three samples as analyzed by each of the four laboratories using the LLNL spike are presented in Table V and Figure I. Normalized minor isotope data have also been included in the table. In the figure, data are plotted both with and without the mass bias correction. Each uncorrected/corrected pair is labeled by the symbol for the laboratory contributing the data. The order of the points from the left is Sample 1, Sample 2, and Sample 3.

For the averages of the multiple analyses performed by the individual laboratories, only one of 12 uncorrected analyses differs by more than $1 \%$ from the assumed value. None of the corrected values differs as much as $1 \%$. In most cases, the bias correction makes only a small difference in the average of the multiple analyses of a single laboratory, but in at least one case for Laboratory $\mathrm{L}$ the correction for a single analysis exceeded $1 \%$ (see Appendix E). This laboratory's mass spectrometer uses voltage rather than magnetic scanning. This results in this laboratory having generally larger and more variable bias corrections.

In 8 of the 12 cases $\left(\sigma_{\text {corr }} / \sigma_{\text {uncorr }}\right.$ in Table $\left.V\right)$, the variance among the individual determinations by a single laboratory was reduced when the double-spike correction was applied. This is particularly apparent in Laboratory L's data for Sample 1 where the 
uncorrected data had a standard deviation of $0.7 \%$. The laboratory-to-laboratory variation decreased slightly for all three samples when the double-spike correction was applied. The fact that this reduction was small reflects the fact that the overall uncertainty was not controlled by the bias. If the assumption about the true isotopic compositions of the samples is correct, the accuracy of 11 of the 12 analyses was improved through the use of the double spike.

As noted above, Laboratory L, used $1 \mathrm{ng}$ of uranium in each of its initial analyses. This resulted in relatively large uncertainties particularly compared to Laboratory I, which used 50 ng "loads". Subsequently Laboratory L reanalyzed the samples using larger sample sizes to reduce the measured uncertainties. Because of the limited amount of the LLNL spike available, Laboratory L used only the IAEA spike for this second set of analyses. As part of the initial study Laboratory L analyzed the three samples with the each of the two double spikes. These measurements provide an illustration of the effect of sample size on precision and an opportunity to perform a consistency check on the two double spikes. The data for the two sets of experiments using the IAEA double spike and the values normalized to the IRMM standard values are presented in Table VI. For each sample, the analysis-to-analysis variation (external precision) is smaller (more precise) in the second experiment where larger samples were used.

For a given sample, the ratio of the bias corrected ${ }^{238} \mathrm{U} /{ }^{235} \mathrm{U}$ 's calculated using the two different spikes is an indicator of the bias between the spikes. For the analyses using the LLNL spike, Laboratory I reported substantially more precise data than the other laboratories. Laboratory I's values also agree with the assumed "true" ratios within $0.1 \%$ for each sample. For these reasons Laboratory I's for the LLNL spike and Laboratory L's values for the IAEA spike will be used to compare the spike - Table VII. Based on these data, it appears that there is a $0.24 \%$ bias in the ${ }^{236} \mathrm{U} /{ }^{233} \mathrm{U}$ between the two double spikes.

\section{Discussion}

The significance of the bias correction depends on several factors including the precision of a single analysis and the mode of data collection. The sample-to-sample variation observed by the various laboratories differs by roughly an order of magnitude. This is mostly a reflection of their using different sample sizes with $50 \mathrm{ng}$ samples giving much more precision than $1 \mathrm{ng}$. On the other hand, sample-to-sample variation can reflect differences in the variability of the bias as in Laboratory L's case. Ultimately, no matter how small the apparent uncertainty is for an analysis, one can not be certain the analysis is accurate to the same level without using the double spike to objectively determine the mass bias because sample matrices will effect the bias unpredictably. This experiment is essentially the least sensitive test of bias variability possible because of the extremely simple sample matrix - evaporated high-purity standard. "Real" samples with more complicated matrices would be expected to show larger variations in mass bias.

When the double spike was used to correct for mass bias, a ${ }^{238} U /{ }^{235} U$ accuracy of better than $1 \%$ was achieved for all of the samples, even for samples as small as $1 \mathrm{ng}$. For $50 \mathrm{ng}$ of uranium, $0.1 \%$ accuracy was achieved. This was the first inter-laboratory comparison 
using the double spike. Operating conditions such as sample size, spiking level, and data reduction routines have not been optimized. For example Laboratory I found that their data reduction routine was artificially inflating uncertainties because of the choice of isotope used in ratio calculations (Appendix B). Switching to an alternative isotope reduced the calculated uncertainties by $30 \%$. With optimization it should be possible to routinely achieve $0.1 \%$ accuracy for $50 \mathrm{ng}$ of uranium using conventional TIMS. (While not part of this study, it should be noted that with the use of the double spike technique $0.01 \%$ accuracy may be obtainable by techniques such as multi-collector inductivelycoupled-plasma mass spectrometry that are just now becoming available.)

Achieving the goal of high accuracy will depend on verifying the isotopic composition of the double spike. At this time it appears that at least one of the two double spikes is biased by a few tenths of a percent. Verification of the isotopic composition of the spikes can be achieved by analyzing mixtures of each double spike and internationally recognized isotopic standards such as U500, which has a well-characterized ${ }^{238} \mathrm{U} /{ }^{235} \mathrm{U}$ near unity. In the absence of such a verification it is more correct to speak of interlaboratory agreement of $0.1 \%$ where all laboratories use the same double spike. The fact that IAEA has recently provided a copious supply of double spike will facilitate optimization and wider application of the double spike technique. 
Table 1

\begin{tabular}{|l|l|l|l|l|}
\hline & $\mathrm{R} 236 / 233$ & $\mathrm{R} 234 / 233$ & $\mathrm{R} 235 / 233$ & $\mathrm{R} 238 / 233$ \\
\hline LLNL double spike & 0.94967 & 0.000179 & 0.000090 & 0.000803 \\
\hline 2 sigma & 0.00018 & 0.000001 & 0.000001 & 0.000002 \\
\hline IAEA double spike* & 0.96507 & 0.00380 & 0.000071 & 0.00057 \\
\hline & & & & \\
\hline
\end{tabular}

* Uncertainty not known.

Table II

Results without double spike correction

\begin{tabular}{|c|c|c|c|c|c|c|}
\hline \multirow[b]{2}{*}{ Sample 1} & \multicolumn{3}{|c|}{ Unspiked } & \multicolumn{3}{|c|}{ Spiked $^{\dagger}$} \\
\hline & $\begin{array}{l}\text { \# of } \\
\text { runs }\end{array}$ & $238 / 235$ & $2 \sigma$ & $\begin{array}{l}\text { \# of } \\
\text { runs }\end{array}$ & $238 / 235$ & $2 \sigma$ \\
\hline Lab I & 5 & 139.74 & 0.33 & 6 & 139.68 & 0.13 \\
\hline Lab J & 2 & 139.49 & 0.17 & 3 & 137.96 & 1.08 \\
\hline Lab K & 2 & 138.39 & 0.04 & 3 & 138.56 & 0.83 \\
\hline Lab L & 2 & 140.23 & $N^{*}$ & 4 & 138.65 & 1.98 \\
\hline Average & & 139.46 & & & 138.71 & \\
\hline $2 \sigma$ & & 1.56 & & & 1.43 & \\
\hline
\end{tabular}

Sample 2

\begin{tabular}{|c|c|c|c|c|c|c|}
\hline Lab I & 2 & 136.69 & 0.26 & 3 & 136.77 & 0.28 \\
\hline Lab J & 2 & 136.32 & 0.08 & 3 & 136.44 & 0.81 \\
\hline Lab K & 2 & 135.66 & 0.38 & 3 & 135.68 & 0.23 \\
\hline Lab L & 2 & 137.78 & ND & 4 & 136.47 & 0.88 \\
\hline Average & & 136.61 & & & 136.34 & \\
\hline $2 \sigma$ & & 1.77 & & & 0.93 & \\
\hline
\end{tabular}

Sample 3

\begin{tabular}{|c|c|c|c|c|c|c|}
\hline Lab I & 4 & 134.10 & 0.34 & 9 & 133.96 & 0.33 \\
\hline Lab J & 2 & 133.80 & 0.94 & 3 & 133.50 & 1.89 \\
\hline Lab K & 2 & 133.06 & 0.30 & 3 & 132.98 & 0.47 \\
\hline Lab L & 2 & 136.74 & ND & 3 & 133.15 & 0.61 \\
\hline Average & & 134.42 & & & 133.40 & \\
\hline $2 \sigma$ & & 3.21 & & & 0.87 & \\
\hline
\end{tabular}

${ }^{\dagger}$ Excludes samples with IAEA spike

* ND = no data reported 


\section{Table III}

Double Spike Corrected Data

$\begin{array}{llllllll}\text { Ave. }^{\dagger} & 2 \sigma & 238 / 235 & 2 \sigma & 236 / 235 & 2 \sigma & 234 / 235 & 2 \sigma \\ \text { Corr. } & & & & \end{array}$

\begin{tabular}{|c|c|c|c|c|c|c|c|c|}
\hline \multicolumn{9}{|c|}{ Sample 1} \\
\hline Lab I & 1.0007 & 0.0018 & 139.77 & 0.30 & 0.00185 & 0.00008 & 0.00758 & 0.00006 \\
\hline Lab J & 1.0066 & 0.0056 & 138.86 & 1.73 & 0.00217 & 0.00018 & 0.00718 & 0.00000 \\
\hline Lab K & 1.0008 & 0.0115 & 138.68 & 0.77 & 0.00169 & 0.00003 & 0.00754 & 0.00021 \\
\hline Lab L & 0.9939 & 0.0177 & 139.50 & 0.75 & 0.00169 & ND & 0.00763 & ND \\
\hline Average & & & 139.20 & & 0.00185 & & 0.00748 & \\
\hline $2 \sigma$ & & & 1.04 & & 0.00045 & & 0.00041 & \\
\hline \multicolumn{9}{|c|}{ Sample 2} \\
\hline Lab I & 1.0010 & 0.0022 & 136.91 & 0.05 & 0.00214 & 0.00003 & 0.00772 & 0.00007 \\
\hline Lab J & 1.0066 & 0.0057 & 137.34 & 0.69 & 0.00218 & 0.00009 & 0.00741 & 0.00019 \\
\hline Lab K & 1.0067 & 0.0034 & 136.59 & 0.12 & 0.00188 & 0.00005 & 0.00762 & 0.00010 \\
\hline Lab L & 1.0006 & 0.0039 & 136.39 & 0.66 & 0.00208 & ND & 0.00763 & ND \\
\hline Average & & & 136.81 & & 0.00207 & & 0.00760 & \\
\hline $2 \sigma$ & & & 0.83 & & 0.00027 & & 0.00026 & \\
\hline \multicolumn{9}{|c|}{ Sample 3} \\
\hline Lab I & 1.0011 & 0.0022 & 134.11 & 0.19 & 0.00215 & 0.00005 & 0.00770 & 0.00005 \\
\hline Lab J & 1.0060 & 0.0021 & 134.29 & 1.62 & 0.00233 & 0.00018 & 0.00779 & 0.00039 \\
\hline Lab K* & 1.0032 & 0.0135 & 133.40 & 1.35 & 0.00200 & 0.00020 & 0.00771 & 0.00003 \\
\hline Lab L & 0.9961 & 0.0088 & 133.68 & 0.79 & 0.00207 & ND & 0.00785 & ND \\
\hline Average & & & 133.87 & & 0.00214 & & 0.00777 & \\
\hline $2 \sigma$ & & & 0.81 & & 0.00028 & & 0.00014 & \\
\hline
\end{tabular}

${ }^{\dagger}$ Ave. Corr. $=$ the average ratio of the fractionation corrected to uncorrected 238/235.

* One 238/235 value excluded - 236/233 was anomalous.

\section{Table IV}

\section{Assumed Composition of Samples}

\begin{tabular}{|c|c|c|c|}
\hline & $\mathbf{2 3 8} / \mathbf{2 3 5}$ & $\mathbf{2 3 6} / \mathbf{2 3 5}$ & $\mathbf{2 3 4} / \mathbf{2 3 5}$ \\
\hline Sample 1 & 139.84 & 0.00169 & 0.00750 \\
\hline Sample 2 & 136.97 & 0.00197 & 0.00759 \\
\hline Sample 3 & 134.23 & 0.00199 & 0.00764 \\
\hline
\end{tabular}


Table V

\section{Normalized Data}

\section{Double-spike Corrected $^{\dagger}$}

$238 / 235 \quad 2$

Sample 1

\section{Uncorrected}

$\overline{238 / 235} 2 \sigma^{*} \quad \frac{\sigma \text { corr }}{\sigma \text { uncorr }}$

$\begin{array}{llllllllll}\text { Lab I } & 0.9995 & 0.0021 & 1.094 & 0.047 & 1.011 & 0.007 & 0.9989 & 0.0009 & 2.32 \\ \text { Lab J } & 0.9930 & 0.0124 & 1.283 & 0.106 & 0.957 & 0.001 & 0.9865 & 0.0077 & 1.60 \\ \text { Lab K } & 0.9917 & 0.0055 & 1.001 & 0.018 & 1.006 & 0.028 & 0.9908 & 0.0059 & 0.93 \\ \text { Lab L } & 0.9976 & 0.0053 & 1.002 & \text { ND } & 1.018 & \text { ND } & 0.9915 & 0.0142 & 0.38 \\ & & & & & & & & & \\ \text { Ave. } & 0.9955 & & 1.095 & & 0.998 & & 0.9919 & & \\ 2 \sigma & 0.0074 & & 0.266 & & 0.055 & & 0.0102 & \end{array}$

Sample 2

$\begin{array}{llllllllll}\text { Lab I } & 0.9996 & 0.0004 & 1.084 & 0.013 & 1.017 & 0.009 & 0.9985 & 0.0020 & 0.18 \\ \text { Lab J } & 1.0027 & 0.0050 & 1.107 & 0.045 & 0.977 & 0.024 & 0.9962 & 0.0059 & 0.85 \\ \text { Lab K } & 0.9972 & 0.0009 & 0.954 & 0.025 & 1.004 & 0.013 & 0.9906 & 0.0017 & 0.52 \\ \text { Lab L } & 0.9958 & 0.0048 & 1.056 & \text { ND } & 1.006 & \text { ND } & 0.9964 & 0.0064 & 0.75 \\ & & & & & & & & & \\ \text { Ave. } & 0.9988 & & 1.050 & & 1.001 & & 0.9954 & & \\ 2 \sigma & 0.0061 & & 0.135 & & 0.035 & & 0.0068 & & \end{array}$

Sample 3

\begin{tabular}{llllllllll} 
Lab I & 0.9991 & 0.0014 & 1.084 & 0.025 & 1.008 & 0.007 & 0.9980 & 0.0024 & 0.59 \\
Lab J & 1.0005 & 0.0121 & 1.172 & 0.091 & 1.021 & 0.052 & 0.9946 & 0.0141 & 0.86 \\
Lab K & 0.9938 & 0.0101 & 1.007 & 0.101 & 1.010 & 0.004 & 0.9907 & 0.0035 & 2.87 \\
Lab L & 0.9959 & 0.0059 & 1.040 & ND & 1.028 & ND & 0.9920 & 0.0045 & 1.29 \\
& & & & & & & & & \\
Ave. & 0.9973 & & 1.076 & & 1.017 & & 0.9938 & \\
$2 \sigma$ & 0.0060 & & 0.142 & & 0.019 & 0.0065 & \\
\multicolumn{7}{l}{ corrected to LLNL spike and normalized to IRMM standards } \\
* standard deviation of individual analyses performed by a single laboratory.
\end{tabular}




\section{Table VI}

\section{Normalized Results with IAEA Double Spike Data from Laboratory $L$}

\begin{tabular}{|c|c|c|c|c|c|}
\hline \multirow[b]{2}{*}{ Sample } & \multirow[b]{2}{*}{ Filament } & \multicolumn{2}{|c|}{ First Experiment } & \multicolumn{2}{|c|}{ Second Experiment ${ }^{*}$} \\
\hline & & $\mathrm{R} 8 / 5$ & Normalized & $\mathrm{R} 8 / 5$ & Normalized \\
\hline 1 & $\begin{array}{l}1 \\
2 \\
3\end{array}$ & $\begin{array}{l}139.74 \\
139.94 \\
140.57\end{array}$ & $\begin{array}{l}0.9993 \\
1.0007 \\
1.0052\end{array}$ & $\begin{array}{l}140.45 \\
139.92 \\
139.98\end{array}$ & $\begin{array}{l}1.0044 \\
1.0006 \\
1.0010\end{array}$ \\
\hline & $\begin{array}{l}\text { Avg } \\
2 \sigma\end{array}$ & $\begin{array}{r}140.08 \\
0.86\end{array}$ & $\begin{array}{l}1.0017 \\
0.0062\end{array}$ & $\begin{array}{r}140.12 \\
0.58\end{array}$ & $\begin{array}{l}1.0020 \\
0.0042\end{array}$ \\
\hline 2 & $\begin{array}{l}1 \\
2 \\
3\end{array}$ & $\begin{array}{l}136.54 \\
137.25 \\
137.12\end{array}$ & $\begin{array}{l}0.9969 \\
1.0021 \\
1.0011\end{array}$ & $\begin{array}{l}137.02 \\
137.27 \\
137.08\end{array}$ & $\begin{array}{l}1.0004 \\
1.0022 \\
1.0008\end{array}$ \\
\hline & $\begin{array}{l}\text { Avg } \\
2 \sigma\end{array}$ & $\begin{array}{r}136.97 \\
0.76\end{array}$ & $\begin{array}{l}1.0000 \\
0.0055\end{array}$ & $\begin{array}{r}137.12 \\
0.26\end{array}$ & $\begin{array}{l}1.0011 \\
0.0019\end{array}$ \\
\hline 3 & $\begin{array}{l}1 \\
2 \\
3\end{array}$ & $\begin{array}{l}132.94 \\
133.39 \\
133.44\end{array}$ & $\begin{array}{l}0.9904 \\
0.9937 \\
0.9941\end{array}$ & $\begin{array}{l}134.43 \\
134.64 \\
134.48\end{array}$ & $\begin{array}{l}1.0015 \\
1.0031 \\
1.0019\end{array}$ \\
\hline & $\begin{array}{l}\text { Avg } \\
2 \sigma\end{array}$ & $\begin{array}{r}133.26 \\
0.55\end{array}$ & $\begin{array}{l}0.9928 \\
0.0041\end{array}$ & $\begin{array}{r}134.52 \\
0.23\end{array}$ & $\begin{array}{l}1.0022 \\
0.0017\end{array}$ \\
\hline
\end{tabular}

Table VII

$\begin{array}{cccccc}\text { Sample } & \text { IAEA } & 2 \sigma & \text { LLNL } & 2 \sigma & \text { IAEA/LLNL } \\ 1 & 1.0020 & 0.0042 & 0.9995 & 0.0021 & 1.0025 \\ 2 & 1.0011 & 0.0019 & 0.9996 & 0.0004 & 1.0015 \\ 3 & 1.0022 & 0.0017 & 0.9991 & 0.0014 & 1.0031 \\ & & & & & \\ \text { avg } & 1.0018 & & 0.9994 & & 1.0024 \\ 2 \text { sd } & 0.0011 & & 0.0005 & & 0.0015\end{array}$


Figure I

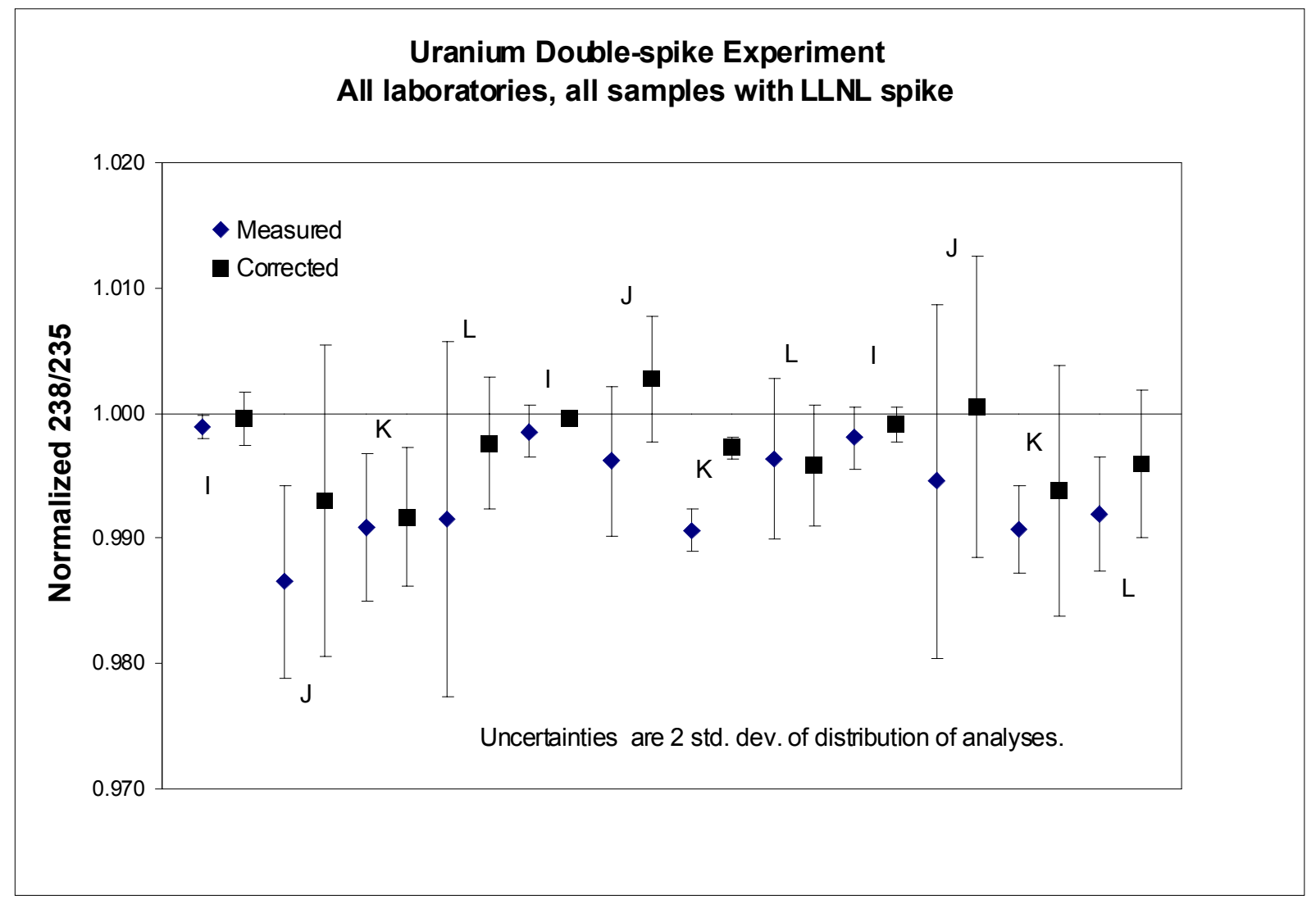




\section{Appendix A}

\section{Double Spike Formalism}

There are a variety of ways to formulate the double spike equations. The purpose of this appendix is to describe one of them and to comment on some subtleties of the double spike technique. This discussion will be specific to uranium but the principles are general. A fuller discussion of this formalism can be found in Hamelin et al. (Geochimica et Cosmochimica Acta 49 (1985) 173.

Three simultaneous equations are used to solve for the mass bias in each the spiked analysis, the mass bias in the unspiked analysis, and the amount of uranium in the sample. These equations are of the form

$\left[(\mathrm{i} / 8)_{\mathrm{M}} *(8 / 3)_{\mathrm{T}}-(\mathrm{i} / 3)_{\mathrm{T}}\right]\{3 \mathrm{t} / 8 \mathrm{u}\}-\left[(\mathrm{i}-8)(\mathrm{i} / 8)_{\mathrm{M}}\right]\left\{\left(1+(8 / 3)_{\mathrm{T}} *(3 \mathrm{t} / 8 \mathrm{u})\right) \mathrm{f}_{\mathrm{s}}\right\}+\left[(\mathrm{i}-8)(\mathrm{i} / 8)_{\mathrm{U}}\right]\left\{\mathrm{f}_{\mathrm{u}}\right\}$ $=(\mathrm{i} / 8)_{\mathrm{U}}-(\mathrm{i} / 8)_{\mathrm{M}}$

where quantities in \{\} are the unknowns and quantities in [ ] are measured or known for the spike. Terms of the form (i/8) are isotopic ratios where $i$, which is fixed in each equation, has the values 3,5 , and 6 and 8 stands for ${ }^{238} \mathrm{U} . \mathrm{M}, \mathrm{T}$, and $\mathrm{U}$ indicate ratios in the mixture, tracer (double spike), and unspiked runs, respectively. $\mathrm{M}$ and $\mathrm{U}$ are measured values. $T$ is the true ratio in the tracer. $f_{u}$ and $f_{s}$ are the fractionations per mass unit in the unspiked and spiked runs. (i-8) is the mass difference between isotope $i$ and 238. $3 \mathrm{t} / 8 \mathrm{u}$ is the ratio of ${ }^{233} \mathrm{U}$ in the tracer to ${ }^{238} \mathrm{U}$ in the sample. The equations are readily solvable for the \{\} 'ed terms by matrix techniques. Having determined the mass bias, one can correct the measured isotopic ratios.

For this experiment, the uranium isotopic ratios were to be calculated with respect to

${ }^{235} \mathrm{U}$. It is tempting to rewrite the matrix equations with ${ }^{235} \mathrm{U}$ instead of ${ }^{238} \mathrm{U}$ as the reference isotope; i.e., the denominator in the ratios. Unless the uranium is highly enriched, doing this will artificially raise the calculated uncertainty in the bias corrected ${ }^{238} \mathrm{U} /{ }^{235} \mathrm{U}$ because $236 / 238$ and $233 / 238$ will have less uncertainty than $236 / 235$ and $233 / 235$. Laboratory I found that the uncertainty on ${ }^{238} \mathrm{U} /{ }^{235} \mathrm{U}$ was $30 \%$ lower for the same data set when the bias corrections were formulated relative to 238 .

The bias is determined using 238 as the reference isotope, but the data are to be reported relative to 235 . Rather than calculating all ratios relative to 238 and then dividing by $235 / 238$, it is desirable to calculate the measured ratios $233 / 235,234 / 235$, and $236 / 235$ directly from the raw data and apply the bias correction for mass bias. This avoids unnecessarily adding the uncertainty associated with $235 / 238$.

Rather than trying to rigorously propagate the uncertainties of the measurements through the double spike equations, one can estimated the uncertainty in the final results by a Monte Carlo method. The terms for the measured values in the matrix equations can be allowed to vary with their individual uncertainty ranges and the results calculated a few 
hundred times. The overall uncertainty can then be estimated from the range of calculated values.

If the double spike is well characterized and composed of nearly pure ${ }^{233} \mathrm{U}$ and ${ }^{236} \mathrm{U}$, one need not be highly concerned about optimal spiking levels. For near natural samples the precision of the 238/235 will be governed by the precision with which 235 , the less abundant isotope, can be measured. As long as the amount of spike added is several times the amount of 235 in the sample, the uncertainty in the bias corrected value will still be governed by the 235 itself. Of course one does not want to add so much spike that the 235 and 238 in the spike become significant corrections to the measured 238/235 in the spiked sample.

Each laboratory was asked to make three measurements of the spiked aliquot and two of the unspiked for each sample. Because only one measurement of each is required to determine the bias corrected isotope ratios, the multiple analyses provide an opportunity to use different combinations of analyses to evaluate the effect of variations in the spiked and unspiked results on the final ratios. Laboratory I made such an evaluation and showed (see Appendix B) that even though the 238/235 reported is calculated from the measured 238/235 in the unspiked sample and $f_{u}$, the result is highly insensitive to which of the unspiked analyses is used for the calculation. Of course, determinations of the minor isotopes, particularly 233 and 236, in the sample depend on the measurements in the unspiked analyses.

In environmental samples ${ }^{233} \mathrm{U}$ and ${ }^{236} \mathrm{U}$ can be expected to be absent or insignificant compared to the spike added. If one is only concerned with ${ }^{238} \mathrm{U} /{ }^{235} \mathrm{U}$, it is possible to skip the unspiked analysis. This can result in nearly a factor of two reduction in cost. 


\section{Appendix B}

\section{Reports from Laboratory I}

(Reformatted to fit page)

\section{Double Spike Roundrobin for NWAL Laboratory I Report}

$10 / 30 / 98$

\section{Introduction and Summary}

Data are on following worksheets.

Numbering system used on subsequent worksheets: $x$ ysn zum where

$\mathrm{x}=$ sample number as supplied on original sample

ys = filament load number - spiked

$\mathrm{n}=$ run number for filament ys

$\mathrm{zu}=$ filament load number - unspiked

$\mathrm{m}=$ run number for filament $z u$

A run is one complete analysis starting with a cold filament.

\section{Sample History:}

Instructions were to load three spiked and two unspiked samples of each sample using $50 \mathrm{ng} \cup$ per load.

Each lab received $2 \mathrm{~mL}$ of LLNL double spike - enough to spike to roughly $3 / 5=$ 4.

Three samples numbered 1 - 3 were received from LANL dried in quartz tubes. Each tube said to contain $750 \mathrm{ng} \mathrm{U}$.

Wes Eford advised that samples dried from $\mathrm{HCl}$ and to rinse bottom 1.5 inches to recover.

Added 200 microL 4N HNO3 to each tube. Shook and rolled on angle to wet sides. Poured liquid into Savillex vials.

Rinsed each tube with 100 microL of acid and poured into vials. Gives concentration of $2.5 \mathrm{ng} / \mathrm{microL}$.

For spiked runs transferred 60 microL to second set of vials. Spiked each sample once and loaded aliquots.

For unspiked runs transferred 40 microL to second set of vials.

Evaporated dry and redissolved in nitric for loading. Loaded fractions of loading acid $1 / 3$ or $1 / 2$ as appropriate.

Used zone-refined, "baked" triple filaments - Ta side, Re center. Dry sample on sides then heat to dull red briefly.

Several loadings did not run. Apparantly the $U$ was not dissolved and most got loaded on one filament.

On the grounds that the sample spot in the vial may have been missed, two additional filaments, each, were loaded for the spiked portion of samples 2 and 3 . These did not run so material was not in vial. 
Additional aliquots were taken from samples 2 and 3 (2 each), separately spiked (4 spikings), and loaded.

One loading of sample 2 never did run well. Others were OK.

If a sample was not exhausted at the end of the "run", it was restarted as a new

run.

Generally a run consisted of 24 accumulations of 10 mass scans each. The mass scans included all isotopes and several background peaks.

After collection the data were reprocessed "offline" to remove outliers and generate the ratios needed for the doublespike calculations.

\section{Spiking:}

The weight difference in the spike between the time it was put in the vial for NWAL distribution and the time it was used, was equivalent to $0.17 \%$ evaporation (6/12/98 - 9/23/98).

Spike weights were tracked along with sample aliquoting. These data are not being reported here but are available.

\section{Blank:}

Each loading was equivalent to 20 microL of evaporated acid plus the loading acid (ca. 2 microL).

Twenty microL of acid was added to a vial along with $73.6 \mathrm{mg}$ of the double spike (0.81 ng 233).

Measured $238 / 233=0.0017$. If all 238 from blank, then loading blank is $1.3 \mathrm{pg}$. If $238 / 233$ in double spike is $8 \mathrm{E}-4$ as advertised, blank is $0.7 \mathrm{pg}$.

These analyses were done in "normal" chemistry labs not a cleanroom.

\section{Double Spike Methodology:}

Data were reduced using simultaneous equations similar to those described by Hamelin et al. (Geochimica 49, 173.)

In our previous work the equations were formulated relative to 235 because data were being reported that way.

This overestimates the uncertainties because of the covariance of 235 in the $236 / 235$ and $233 / 235$ ratios.

Ideally the equations would be in terms of measured 236/233, but I have not been able to solve these equations except by omitting certain minor terms. For this work the equations were formulated in terms of 238 . This reduced the calculated uncertainty on $238 / 235$ by ca. $30 \%$.

For the minor isotopes, ratios relative to 235 were calculated directly in the offline reduction of the raw data.

Uncertainties were estimated by a Monte Carlo method where the individual uncertainites on the terms in the equations were allowed to vary with their individual uncertainties. 200 iterations were used. 
The three simultaneous equations used to correct for fractionation were of the form

$\left[(\mathrm{i} / 8) \mathrm{M}^{*}(8 / 3) \mathrm{T}-(\mathrm{i} / 3) \mathrm{T}\right]\{3 \mathrm{t} / 8 \mathrm{u}\}-[(\mathrm{i}-8)(\mathrm{i} / 8) \mathrm{M}]\left\{\left(1+(8 / 3) \mathrm{T}^{*}(3 \mathrm{t} / 8 \mathrm{u})\right) \mathrm{fs}\right\}+[(\mathrm{i}-8)(\mathrm{i} / 8) \mathrm{U}]\{\mathrm{fu}\}$ $=(\mathrm{i} / 8) \mathrm{U}-(\mathrm{i} / 8) \mathrm{M}$

where quantities in \{\} are the unknowns and quantities in [ ] are measured or known for the spike terms of the form (i/8) are isotopic ratios where $i=3,5$, and 6 $\mathrm{M}, \mathrm{T}$, and $\mathrm{U}$ indicate ratios in the mixture, tracer (double spike), and unspiked runs

fu and fs are the fractionations per mass unit in the unspiked and spiked runs

(i-8) is the mass difference between isotope $i$ and 238

\section{Results Summary}

Details are on following sheets. The numbers below are the averages of the individual runs and represent my best estimate of the true values and overall uncertainties.

\begin{tabular}{lrllr} 
Sample & $238 / 235$ & \multicolumn{2}{c}{$236 / 235$} & \multicolumn{2}{c}{$234 / 235$} & $233 / 235$ \\
& & & & \\
Sample 1 & 139.77 & 0.00185 & 0.00758 & -0.00001 \\
2 sigma & 0.30 & 0.00008 & 0.00006 & 0.00002 \\
2 sig \% & 0.21 & 4 & 0.7 & -214 \\
Sample 2 & 136.91 & 0.00214 & 0.00772 & 0.00000 \\
2 sigma & 0.05 & 0.00003 & 0.00007 & 0.00002 \\
2 sig \% & 0.04 & 1 & 0.9 & 385 \\
Sample 3 & 134.11 & 0.00215 & 0.00770 & -0.00001 \\
2 sigma & 0.19 & 0.00005 & 0.00005 & 0.00005 \\
2 sig \% & 0.14 & 2 & 0.7 & -372
\end{tabular}

Notes: The 236 is roughly proportional to $235 / 238$ while $234 / 235$ is roughly constant.

The data are not sufficiently precise to determine whether the samples are a mixture of 2 components.

No 233 was observed.

The ratio of 238/235 in the unspiked samples to the ratio in the spiked samples can be used to show that the spike makes no significant contribution to 238/235. Similarly the ratio of $238 / 235$ in the spiked runs uncorrected for fractionation to the fractionation corrected ratio shows the extent of fractionation. 


\begin{tabular}{|c|c|c|c|}
\hline $238 / 235$ & Spiked/Unspiked & $\begin{array}{l}\text { Spiked } \\
\text { uncorr/Fract corr. }\end{array}$ & \\
\hline Sample 1 & 1.000449 & 0.999329 & \\
\hline ample 2 & 0.999414 & 0.998983 & $\begin{array}{l}\text { These data are the averages for the } \\
\text { multiple analyses not individual runs }\end{array}$ \\
\hline$m$ & 1.000998 & 0.998 & \\
\hline
\end{tabular}

For this data set, mass fractionation was essentially negligible and within the uncertainties of the analyses.

However, for two of the three samples, the std.dev. of the fractionation corrected values is less than that of the uncorrected values.

In Sample 1 the distribution of 238/235 values is unusually "tight".

\section{Fractionation Corrected Data}

\section{Lab I}

\section{Vary spiked run for a single unspiked run.}

Notes: $\quad 238 / 235$ insensitive to choice of unspiked run so only one unspiked run analyzed below.

Minor isotope uncertainties must be estimated from varying choice of unspiked runs.

$\begin{array}{lrrrrrrrr}\text { Sample } & \text { R8/5 } & \text { 2 s.d. } & \text { R6/5 } & \text { 2 s.d. } & \text { R4/5 } & \text { 2 s.d. } & \text { R3/5 } & \text { 2 s.d. } \\ & & & & & & & & \\ \text { 3 5sb 1ua } & 133.92 & 0.21 & 0.00214 & 0.00005 & 0.00767 & 0.00005 & -0.00002 & 0.00002 \\ \text { 3 5sa 1ua } & 134.02 & 0.21 & 0.00214 & 0.00005 & 0.00767 & 0.00005 & -0.00002 & 0.00002 \\ \text { 3 4sc 1ua } & 134.24 & 0.18 & 0.00214 & 0.00005 & 0.00767 & 0.00005 & -0.00002 & 0.00002 \\ \text { 3 4sb 1ua } & 134.17 & 0.18 & 0.00214 & 0.00005 & 0.00767 & 0.00005 & -0.00002 & 0.00002 \\ \text { 3 4sa 1ua } & 134.15 & 0.21 & 0.00214 & 0.00005 & 0.00767 & 0.00005 & -0.00002 & 0.00002 \\ \text { 3 3sb 1ua } & 134.14 & 0.17 & 0.00214 & 0.00005 & 0.00767 & 0.00005 & -0.00002 & 0.00002 \\ \text { 3 3sa 1ua } & 134.18 & 0.19 & 0.00214 & 0.00005 & 0.00767 & 0.00005 & -0.00002 & 0.00002 \\ \text { 3 2s 1ua } & 134.06 & 0.40 & 0.00214 & 0.00005 & 0.00767 & 0.00005 & -0.00002 & 0.00002 \\ \text { 3 1s 1ua } & 134.11 & 0.31 & 0.00214 & 0.00005 & 0.00767 & 0.00005 & -0.00002 & 0.00002 \\ & & & & & & & & \\ \text { Ave. } & 134.11 & & & & & & & \\ \text { 2 s.d. } & 0.19 & & & & & & & \\ \text { 2 s.d.\% } & 0.14 & & & & & & & \\ & & & & & & & & \\ \text { 2 3sc 2u } & 136.88 & 0.18 & 0.00215 & 0.00005 & 0.00775 & 0.00007 & 0.00001 & 0.00004 \\ \text { 2 3sb 2u } & 136.91 & 0.18 & 0.00215 & 0.00005 & 0.00774 & 0.00007 & 0.00001 & 0.00004 \\ \text { 2 2s 2u } & 136.93 & 0.23 & 0.00215 & 0.00005 & 0.00774 & 0.00007 & 0.00001 & 0.00004 \\ & & & & & & & & \\ \text { Ave. } & 136.91 & & & & & & & \\ \text { 2 s.d. } & 0.05 & & & & & & & \\ \text { 2 s.d.\% } & 0.04 & & & & & & & \end{array}$




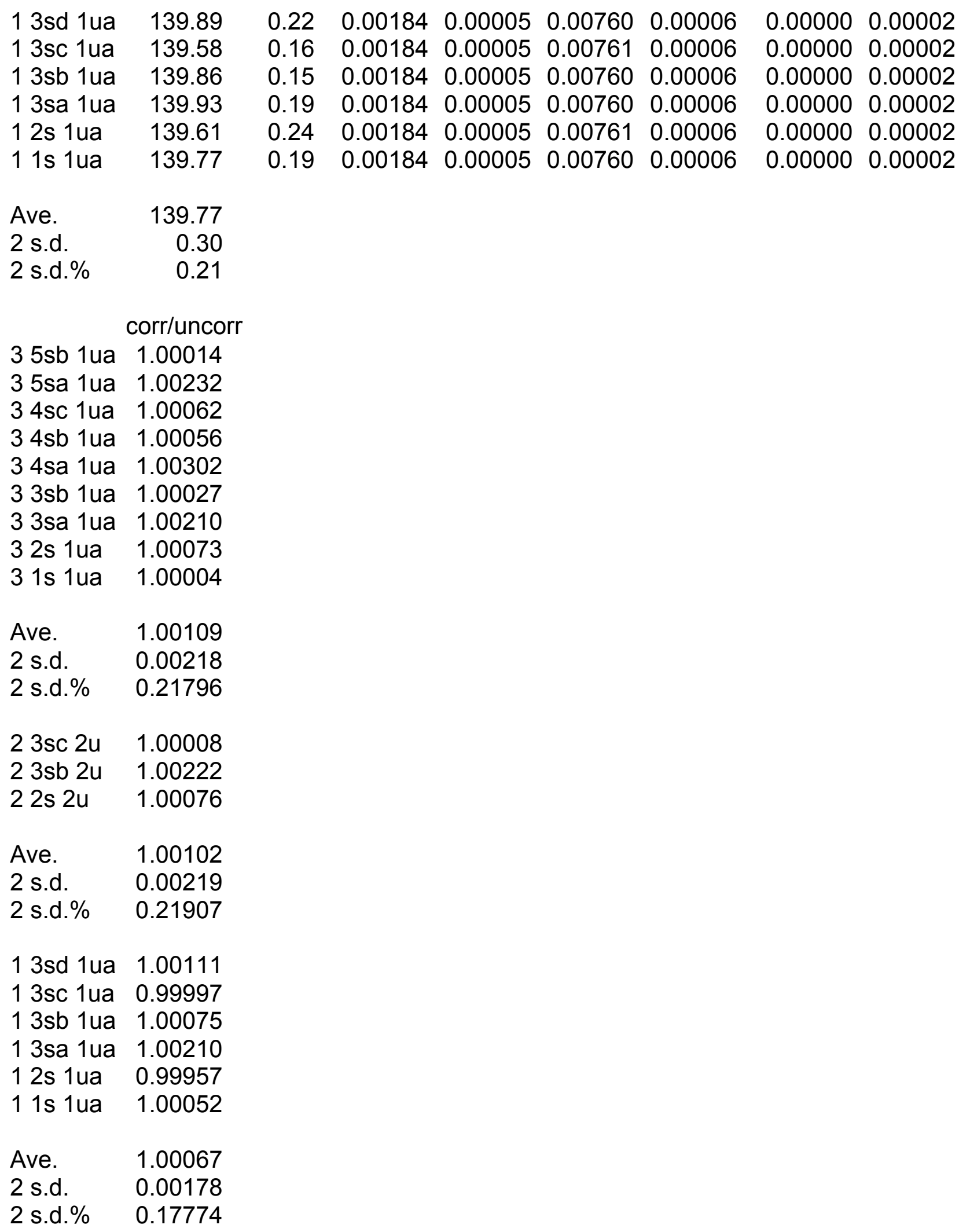




\section{Fractionation Corrected Data Continued}

Vary unspiked run for a single spiked run. (Representative examples.)

Notes: Demonstration that $238 / 235$ is insensitive to choice of unspiked run.

Minor ratios are insensitive to choice of spiked run (see sample 3 data).

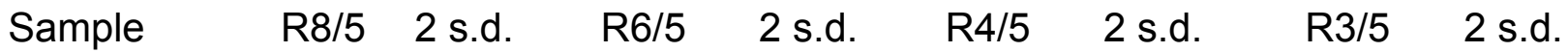

3 4sa 1ua 134.145

3 4sa 1 ub 134.146

3 4sa 2ua 134.146

3 4sa 2ub 134.146

Ave.

2 s.d.

2 s.d.\%

2 2s $1 \mathrm{u} \quad 136.929$

2 2s $2 \mathrm{u} \quad 136.929$

Ave.

2 s.d.

2 s.d.\%

1 3sa 2uc 139.937

1 3sa 2ua 139.934

1 3sa 1ua 139.935

1 3sa 2ub 139.936

1 3sa 1ub 139.934

Ave.

2 s.d.

2 s.d.\%

$12 \mathrm{~s} 2 \mathrm{uc}$

$12 \mathrm{~s} 2 \mathrm{ub}$

12 s $2 u a$

$12 \mathrm{~s} 1 \mathrm{ub}$

Ave.

2 s.d.

2 s.d. $\%$

$11 \mathrm{~s} 2 \mathrm{uc} \quad 139.769$

1 1s $2 \mathrm{ub} \quad 139.768$ $\begin{array}{ll}0.21 & 0.00214\end{array}$

$\begin{array}{lll}0.21 & 0.00217\end{array}$

$\begin{array}{ll}0.21 & 0.00213\end{array}$

$\begin{array}{lll}0.21 & 0.00218\end{array}$

0.00005

0.00767

0.00005

$\begin{array}{lll}-0.00002 & 0.00002\end{array}$

$\begin{array}{ll}0.00003 & 0.00771\end{array}$

0.00006

0.00001

0.00773

0.00007

$-0.00005$

0.00003

$\begin{array}{lll}0.00006 & 0.00771\end{array}$

0.00006

0.00215

0.00770

$-0.00001$

0.00005

0.00005

0.00005

2.3

0.7

$-372$

$\begin{array}{llll}0.23 & 0.00213 & 0.00005\end{array}$

0.00770

0.00006

$0.00000 \quad 0.00002$

$\begin{array}{llll}0.23 & 0.00215 & 0.00005\end{array}$

$\begin{array}{lll}0.00774 & 0.00007\end{array}$

0.000010 .00004

0.00214

0.00772

0.00000

0.00003

0.00007

0.9

0.00002

385 $\begin{array}{llll}0.19 & 0.00191 & 0.00005 & 0.0\end{array}$

$\begin{array}{lll}0.19 & 0.00183 & 0.00003\end{array}$

$\begin{array}{lll}0.19 & 0.00184\end{array}$

0.00005

0.190 .00187

0.00004

0.190 .00180

0.00004

0.00756

0.00750

0.00760

0.00759

0.00754

0.00004

0.00005

0.00006

0.00005

0.00007

0.00185

0.00756

0.00008

1.1

4.4

$0.24 \quad 0.00191$

0.00005

0.00756

0.00004

$\begin{array}{ll}0.24 & 0.00187\end{array}$

0.00004

0.00760

0.00005

0.00005

0.00751

0.00007

$0.24 \quad 0.00180$

0.00003

0.00754

0.00755

0.00008

1.0
0.00185
0.00009

5.1
$-0.00001$

0.00003

$-252$

0.000010 .00003

$0.00000 \quad 0.00004$

$0.00000 \quad 0.00002$

$\begin{array}{lll}0.00000 & 0.00002\end{array}$

$\begin{array}{lll}-0.00003 & 0.00003\end{array}$

$-0.000010 .00003$

$0.00000 \quad 0.00002$

$0.00000 \quad 0.00004$

$-0.000030 .00003$

$-0.00001$

0.00003

$-239$

$\begin{array}{lllllll}0.19 & 0.00191 & 0.00005 & 0.00756 & 0.00004 & -0.00001 & 0.00003\end{array}$ $\begin{array}{lllllll}0.19 & 0.00187 & 0.00004 & 0.00760 & 0.00005 & 0.00000 & 0.00002\end{array}$ 
11 s 2 ua

$11 \mathrm{~s} 1 \mathrm{ub}$

1 1s 1 ua

Ave.

2 s.d.

2 s.d.\%
139.767

139.766

139.767 $\begin{array}{lll}0.19 & 0.00184 & 0.00005\end{array}$

$\begin{array}{llll}0.19 & 0.00180 & 0.00004 & 0.0\end{array}$

$\begin{array}{lllll}0.19 & 0.00184 & 0.00005 & 0.00760\end{array}$

0.00185

0.00008

4.3
0.00758

0.00006

0.7 $\begin{array}{lll}0.00006 & 0.00000 & 0.00002\end{array}$

$\begin{array}{llll}0.00007 & -0.00003 & 0.00003\end{array}$

$\begin{array}{lll}0.00006 & 0.00000 & 0.00002\end{array}$
$-0.00001$

0.00002

$-214$ 


\section{Data Without Fractionation Correction}

\section{Spiked}

\begin{tabular}{|c|c|c|c|c|c|c|c|c|c|c|c|c|}
\hline Sample & $\mathrm{R} 5 / 8$ & 2 sigma & $\mathrm{R} 6 / 8$ & 2 sigma & $\mathrm{R} 4 / 5$ & 2 sigma & $\mathrm{R} 3 / 8$ & 2 sigma & $\mathrm{R} 6 / 3$ & 2 sigma & \multicolumn{2}{|c|}{$\mathrm{R} 8 / 52$ sigma } \\
\hline $35 \mathrm{sb}$ & 0.007468 & 0.000010 & 0.02041 & 0.00001 & 0.00821 & 0.00008 & 0.02148 & 0.00001 & 0.9508 & 0.0008 & 133.90 & 0.17 \\
\hline $35 s a$ & 0.007479 & 0.000008 & 0.02044 & 0.00001 & 0.00831 & 0.00005 & 0.02156 & 0.00002 & 0.9484 & 0.0010 & 133.71 & 0.14 \\
\hline $34 s c$ & 0.007454 & 0.000007 & 0.02033 & 0.00001 & 0.00825 & 0.00005 & 0.02140 & 0.00001 & 0.9497 & 0.0010 & 134.15 & 0.13 \\
\hline $34 \mathrm{sb}$ & 0.007458 & 0.000007 & 0.02035 & 0.00001 & 0.00823 & 0.00008 & 0.02142 & 0.00001 & 0.9500 & 0.0006 & 134.09 & 0.13 \\
\hline $34 \mathrm{sa}$ & 0.007477 & 0.000006 & 0.02035 & 0.00001 & 0.00824 & 0.00006 & 0.02147 & 0.00003 & 0.9477 & 0.0009 & 133.74 & 0.11 \\
\hline $33 \mathrm{sb}$ & 0.007457 & 0.000007 & 0.02683 & 0.00002 & 0.00845 & 0.00069 & 0.02824 & 0.00002 & 0.9500 & 0.0008 & 134.10 & 0.12 \\
\hline 3 3sa & 0.007468 & 0.000007 & 0.02684 & 0.00002 & 0.00840 & 0.00008 & 0.02830 & 0.00002 & 0.9487 & 0.1 & 133.90 & 0.13 \\
\hline $32 \mathrm{~s}$ & 0.007465 & 0.000017 & 0.02682 & 0.00004 & 0.00845 & 0.00017 & 0.02824 & 0.00003 & 0.9509 & 0.0020 & 133.96 & 0.30 \\
\hline $31 \mathrm{~s}$ & 0.007457 & 0.000013 & 0.02686 & 0.00003 & 0.00806 & 0.00020 & 0.02826 & 0.00002 & 0.9508 & 0.0011 & 134.11 & 0.24 \\
\hline Ave. & 0.007465 & & & & & & & & 0.9497 & & 133.96 & \\
\hline $2 \mathrm{s.d}$ & 0.000018 & & & & & & & & 0.0023 & & 0.33 & \\
\hline 2 s.d. $\%$ & 0.24 & & & & & & & & 0.24 & & 0.24 & \\
\hline $23 \mathrm{sc}$ & .007306 & 0.00 & 0.02858 & 0.000 & 0.00839 & 0.00006 & 0.03007 & 0.00002 & 0.9503 & 0.0006 & 136.87 & 0.14 \\
\hline $23 s$ & 0.007320 & 0.000007 & 0.02860 & 0.00001 & 0.00839 & 0.00005 & 0.03016 & 0.00003 & 0.9482 & 0.0008 & 136.61 & 0.13 \\
\hline $22 \mathrm{~s}$ & 0.007309 & 0.000010 & 0.02872 & 0.00002 & 0.00846 & 0.00006 & 0.03024 & 0.00002 & 0.9497 & 0.0009 & 136.83 & 0.18 \\
\hline Ave & 0.007312 & & & & & & & & 0.9494 & & 136.77 & \\
\hline 2 s.d. & 0.000015 & & & & & & & & 0.0021 & & 0.28 & \\
\hline 2 s.d. $\%$ & 0.20 & & & & & & & & 0.22 & & 0.20 & \\
\hline $13 s d$ & 0.007 & & 0.02 & & & & 0.02920 & & & & & 0.18 \\
\hline 13 & 0.007164 & 0.00 & 0.02772 & 0.0 & 0.0 & 0.00 & 0.02916 & 0.0 & 0.9505 & & 139.58 & 0.13 \\
\hline $13 \mathrm{sb}$ & 0.007155 & 0.000005 & 0.02772 & 0.00001 & 0.00827 & 0.00005 & 0.02918 & 0.00002 & 0.9494 & 0.0006 & 139.76 & 0.10 \\
\hline
\end{tabular}




\begin{tabular}{|c|c|c|c|c|c|c|c|c|c|c|c|}
\hline $13 \mathrm{sa}$ & 0.0071610 .000006 & 0.02774 & 0.00001 & 0.00826 & 0.00007 & 0.02924 & 0.00003 & 0.9482 & 0.0008 & 139.64 & 0.12 \\
\hline $12 s$ & 0.0071600 .000010 & 0.02771 & 0.00001 & 0.00832 & 0.00006 & 0.02914 & 0.00002 & 0.9509 & 0.0005 & 139.67 & 0.19 \\
\hline- & 0.0071590 .000007 & 0.02773 & 0.00002 & 0.00830 & 0.00007 & 0.02919 & 0.00002 & 0.9499 & 0.0005 & 139.69 & 0.13 \\
\hline & 0.00715 & 0.02772 & & 0.00828 & & 0.02919 & & 0.9 & & 139.68 & \\
\hline 50 & 0.000007 & 0.00002 & & 0.00005 & & 0.00007 & & 0.0018 & & 0.13 & \\
\hline d. $\%$ & 0.09 & 0.06 & & 0.6 & & 0.2 & & 0.19 & & 0.09 & \\
\hline
\end{tabular}

Where 236, 234, and 233 averages are not calculated, samples 2 and 3, separate spikings were used so the average and deviation are meaningless. 


\section{Data Without Fractionation Correction Continued}

\section{Unspiked}

\begin{tabular}{|c|c|c|c|c|c|c|c|c|c|c|c|c|c|c|}
\hline iample & $\mathrm{R} 5 / 8$ & 2 sigma & $\mathrm{R} 6 / 8$ & 2 sigma & $\mathrm{R} 4 / 5$ & 2 sigma & $\mathrm{R} 3 / 8$ & 2 sigma & $\mathrm{R} 6 / 5$ & 2 sigma & $\mathrm{R} 3 / 5$ & 2 sigma & $\mathrm{R} 8 / 5$ & 2 sig. \\
\hline 31 ua & 0.007458 & 0.000007 & 0.0000160 & 0.0000004 & 0.00767 & 0.00006 & -0.0000001 & 0.0000002 & 0.00214 & 0.00005 & -0.00002 & 0.00002 & 134.09 & 0.13 \\
\hline $31 \mathrm{ub}$ & 0.007444 & 0.000007 & 0.0000162 & 0.0000002 & 0.00771 & 0.00007 & 0.0000000 & 0.0000002 & 0.00217 & 0.00003 & 0.00001 & 0.00003 & 134.34 & 0.13 \\
\hline 3 2ua & 0.007465 & 0.000008 & 0.0000159 & 0.0000002 & 0.00773 & 0.00007 & -0.0000004 & 0.0000002 & 0.00212 & 0.00003 & -0.00005 & 0.00002 & 133.96 & 0.14 \\
\hline $32 u b$ & 0.007463 & 0.000006 & 0.0000162 & 0.0000004 & 0.00771 & 0.00006 & 0.0000000 & 0.0000001 & 0.00218 & 0.00006 & 0.00001 & 0.00002 & 134.00 & 0.11 \\
\hline Ave. & 0.007457 & & 0.0000161 & & 0.00770 & & -0.0000001 & & 0.00215 & & -0.00001 & & 134.10 & \\
\hline 2 s.d. & 0.000019 & & 0.0000003 & & 0.00005 & & 0.0000004 & & 0.00005 & & 0.00005 & & 0.34 & \\
\hline 2 s.d. $\%$ & 0.25 & & 2 & & 0.7 & & -303 & & 2 & & -372 & & 0.25 & \\
\hline $22 u$ & 0.007321 & 0.000005 & 0.0000157 & 0.0000004 & 0.00775 & 0.00007 & 0.0000001 & 0.0000003 & 0.00215 & 0.00005 & 0.00001 & 0.00004 & 136.60 & 0.10 \\
\hline $21 \mathrm{u}$ & 0.007311 & 0.000008 & 0.0000156 & 0.0000004 & 0.00770 & 0.00006 & 0.0000000 & 0.0000002 & 0.00213 & 0.00005 & 0.00000 & 0.00002 & 136.78 & 0.15 \\
\hline Ave. & 0.007316 & & 0.0000157 & & 0.00772 & & 0.0000001 & & 0.00214 & & 0.000005 & & 136.69 & \\
\hline 2 s.d. & 0.000014 & & 0.0000001 & & 0.00007 & & 0.0000001 & & 0.00002 & & 0.000018 & & 0.26 & \\
\hline 2 s.d. $\%$ & 0.19 & & 0.9 & & 1.0 & & 283 & & 1 & & 385 & & 0.19 & \\
\hline 11 ua & 0.007163 & 0.000007 & 0.0000132 & 0.0000003 & 0.00761 & 0.00006 & 0.0000000 & 0.0000001 & 0.00184 & 0.00005 & 0.00000 & 0.00002 & 139.60 & 0.14 \\
\hline $12 u c$ & 0.007150 & 0.000008 & 0.0000136 & 0.0000003 & 0.00756 & 0.00005 & -0.0000001 & 0.0000002 & 0.00191 & 0.00005 & -0.00001 & 0.00003 & 139.86 & 0.16 \\
\hline 12 ua & 0.007167 & 0.000007 & 0.0000131 & 0.0000002 & 0.00751 & 0.00005 & 0.0000000 & 0.0000003 & 0.00183 & 0.00003 & 0.00000 & 0.00004 & 139.53 & 0.13 \\
\hline $11 \mathrm{ub}$ & 0.007150 & 0.000007 & 0.0000129 & 0.0000003 & 0.00754 & 0.00007 & -0.0000002 & 0.0000002 & 0.00180 & 0.00004 & -0.00003 & 0.00003 & 139.85 & 0.14 \\
\hline $12 u b$ & 0.007150 & 0.000006 & 0.0000134 & 0.0000002 & 0.00760 & 0.00005 & 0.0000000 & 0.0000002 & 0.00187 & 0.00003 & 0.00000 & 0.00002 & 139.87 & 0.11 \\
\hline Ave. & 0.007156 & & 0.000013 & & 0.00756 & & -0.0000001 & & 0.00185 & & -0.000011 & & 139.74 & \\
\hline 2 s.d. & 0.000017 & & 0.000001 & & 0.00008 & & 0.0000002 & & 0.00008 & & 0.000027 & & 0.33 & \\
\hline 2 s.d. $\%$ & 0.23 & & 4 & & 1.1 & & -298 & & 4 & & -252 & & 0.23 & \\
\hline
\end{tabular}



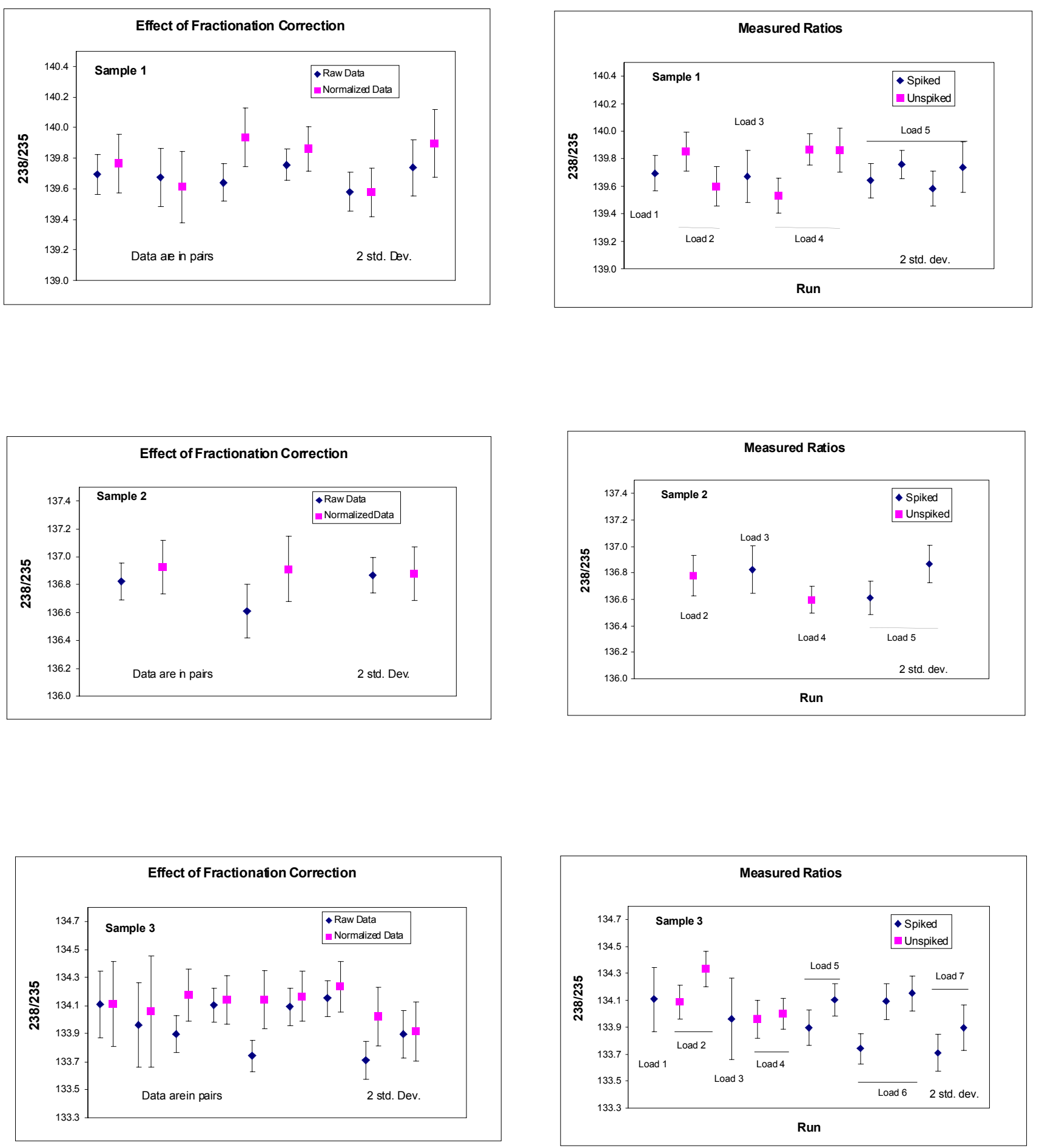


\section{Appendix C}

\section{Reports from Laboratory $\mathbf{J}$}

\section{Reformatted to fit page and editorial comments added (italics)}

\begin{tabular}{|c|c|c|c|c|c|}
\hline sample & \multicolumn{5}{|c|}{$s t d=2$ std deviations } \\
\hline IAEA-A4 & no spk, bkg corr & 139.55 & 0.21 & 0.00204 & 0.00014 \\
\hline IAEA-A5 & no spk, bkg corr & 139.43 & 0.22 & 0.00230 & 0.00014 \\
\hline IAEA-B5 & no spk, bkg corr & 136.34 & 0.14 & 0.00212 & 0.00014 \\
\hline IAEA-B6 & no spk, bkg corr & 136.29 & 0.22 & 0.00225 & 0.00027 \\
\hline IAEA-C4 & no spk, bkg corr & 133.46 & 0.15 & 0.00220 & 0.00013 \\
\hline IAEA-C5 & no spk, bkg corr & 134.13 & 0.43 & 0.00246 & 0.00027 \\
\hline sample & & $\mathrm{R} 8 / 5$ & std & $\mathrm{R} 6 / 5$ & std \\
\hline IAEA-A1 & d. spk, bkg corr & 137.55 & 0.22 & 3.9108 & 0.0083 \\
\hline IAEA-A8 & d. spk, bkg corr & 137.75 & 0.86 & 3.6388 & 0.0309 \\
\hline IAEA-A9 & d. spk, bkg corr & 138.57 & 0.23 & 3.5799 & 0.0083 \\
\hline IAEA-B1 & d. spk, bkg corr & 136.91 & 0.26 & 3.7617 & 0.0096 \\
\hline IAEA-B2 & d. spk, bkg corr & 136.18 & 0.22 & 3.7934 & 0.0081 \\
\hline IAEA-B3 & d. spk, bkg corr & 136.24 & 0.15 & 4.9351 & 0.0070 \\
\hline IAEA-C1 & d. spk, bkg corr & 133.13 & 0.16 & 2.6877 & 0.0046 \\
\hline IAEA-C2 & d. spk, bkg corr & 134.57 & 0.25 & 3.0633 & 0.0081 \\
\hline IAEA-C3 & d. spk, bkg corr & 132.79 & 0.17 & 3.3758 & 0.0060 \\
\hline sample & \multicolumn{4}{|c|}{$\mathrm{R} 8 / 5$} & $\begin{array}{l}\text { AVG } \\
\text { R8/5 }\end{array}$ \\
\hline \multicolumn{6}{|c|}{ Simple double spike correction without cross terms. } \\
\hline IAEA-A1 & ds corrected & 138.35 & & & \\
\hline IAEA-A8 & ds corrected & 138.14 & & IAEA-A & 139.04 \\
\hline IAEA-A9 & ds corrected & 139.74 & & IAEA-B & 136.86 \\
\hline IAEA-B1 & ds corrected & 137.44 & & IAEA-C & 134.02 \\
\hline IAEA-B2 & ds corrected & 137.40 & & & \\
\hline IAEA-B3 & ds corrected & 136.83 & & & \\
\hline IAEA-C1 & ds corrected & 133.87 & & & \\
\hline IAEA-C2 & ds corrected & 135.07 & & & \\
\hline IAEA-C3 & ds corrected & 133.54 & & & \\
\hline
\end{tabular}




\section{Laboratory J's data processed through Appendix B formalism}

Double spike corrected, spiked analyses

$\begin{array}{ccc} & \text { R8/5 } & \text { 2 sig } \\ \text { IAEA-A1 } & 138.47 & 0.47 \\ \text { IAEA-A8 } & 138.26 & 1.87 \\ \text { IAEA-A9 } & 139.85 & 0.51 \\ \text { IAEA-B1 } & 137.56 & 0.53 \\ \text { IAEA-B2 } & 137.52 & 0.44 \\ \text { IAEA-B3 } & 136.94 & 0.30 \\ \text { IAEA-C1 } & 134.01 & 0.36 \\ \text { IAEA-C2 } & 135.21 & 0.56 \\ \text { IAEA-C3 } & 133.66 & 0.38\end{array}$

Average for each sample

$\begin{array}{lrrr} & \mathbf{R} 8 / \mathbf{5} & \mathbf{2} \text { sig } & \text { corr/uncorr } \\ \text { A } & 138.86 & 1.73 & 1.0066 \\ \text { B } & 137.34 & 0.69 & 1.0066 \\ \text { C } & 134.29 & 1.62 & 1.0060\end{array}$




\section{Appendix D}

\section{Reports from Laboratory $\mathrm{K}$}

(Editor's note: “IAEA/LLNL” in Laboratory K's reports refers to the LLNL double spike not the one supplied by IAEA.)

Comments on analyses using LLNL double spike:

Analyses were conducted using the second shipment of 3 samples sent from LANL, since the first shipment was considered compromised, i.e., the Parafilm had become loose and cracked on one of the glass tubes, and all 3 glass tubes had been shipped in a single plastic bag. The sides of each tube were thoroughly rinsed down with $\mathrm{HCl}$, and filled to near the top, to recover all the dried sample, and weighed. Two weighed volumes were taken from each to provide about $20 \mathrm{ng}$ for analysis. A weighed volume was taken from the LLNL double spike to provide about $200 \mathrm{pg}$ for analysis and added to one of the sample aliquots. The aliquot amounts were designed to yield about 40-100K cts/scan at masses 233 and 236, and approximately $2 \mathrm{M}$ cps for ${ }^{238} \mathrm{U}$ in each scan, for good counting statistics and to avoid sample memory in the mass spec source.

The sample+spike aliquot mixtures were subjected to successive evaporation and concentration steps during which beads of anion exchange resin were equilibrated.

Aproximately $5 \%$ of the $U$ is taken up by each bead, averaging $10 \mathrm{pg}$ (total $U$ ) of spike and 1 ng of sample $U$ in each. Two beads were added to the unspiked samples, and three added to the spike samples to provide the number of analyses requested. Single beads were loaded onto carburized Re filaments for analysis on a 3-stage mass spectrometer equipped with ion pulse counting detection capability. Blanks prepared during aliquotting and during bead equilibration and loading were insignificant.

Using the IAEA/LLNL ${ }^{236} \mathrm{U} /{ }^{233} \mathrm{U}$ internal standard to correct for isotopic fractionation, ${ }^{238} \mathrm{U} /{ }^{235} \mathrm{U}$ atom ratio errors are about $1.6 \mathrm{ppt}$. This is to be expected since this method requires the measurement of two atom ratios. To achieve ppt precision using this method, ${ }^{238} \mathrm{U} /{ }^{235} \mathrm{U}$ and ${ }^{236} \mathrm{U} /{ }^{233} \mathrm{U}$ atom ratios must be measured with a precision of about $0.7 \mathrm{ppt}$. The systematic uncertainty associated with our ${ }^{236} \mathrm{U} /{ }^{233} \mathrm{U}$ atom ratio measurements is reflected in observed standard deviations of replicate ${ }^{238} \mathrm{U} /{ }^{235} \mathrm{U}$ atom ratio measurements ranging as high as $16 \mathrm{ppt}$ (i.e., IAEA-2), though we usually obtain better than this. 
IAEA/NWAL SAMPLE SET OF 13OCT1998: IAEA/NWAL Sample No. IAEA-1 CORRECTED FOR ISOTOPIC FRACTIONATION USING INTERNAL ${ }^{236} \mathrm{U} /{ }^{233} \mathrm{U}$ STANDARD IAEA/LLNL

\begin{tabular}{|c|c|c|c|c|c|c|c|c|}
\hline & \multicolumn{6}{|c|}{ SAMPLE ISOTOPIC COMPOSITION } & \multirow{2}{*}{\multicolumn{2}{|c|}{ INTERNAL STD. }} \\
\hline PNNL No. & ${ }^{234} \mathrm{U} /{ }^{235} \mathrm{U}$ & 10 Frror & ${ }^{238} \mathrm{U} / 235 \mathrm{U}$ & $1-\operatorname{Lrman}$ & $236 \mathrm{~J} / 235 \mathrm{U}$ & $1 \sim$ Drror & & \\
\hline & & & & & & & & \\
\hline & & & & & & & & \\
\hline 81455 & 0.007575 & 0.000044 & 138.27 & 0.24 & & & & \\
\hline 81456 & 0.007614 & 0.000049 & 138.73 & 0.23 & & & & \\
\hline 81457 & 0.007417 & 0.000068 & 139.04 & 0.22 & & & & \\
\hline \multirow{2}{*}{ Report: } & 0.007559 & 0.000040 & 13870 & 0.22 & & & 0.94967 & 000018 \\
\hline & & & & & & & \multicolumn{2}{|c|}{ (Above Value is Given) } \\
\hline
\end{tabular}

IAEA/NWAL SAMPLE SET OF 13OCT1998: IAEA/NWAL Sample No. IAEA-1

\section{NOT CORRECTED FOR ISOTOPIC FRACTIONATION}

\begin{tabular}{|c|c|c|c|c|c|c|c|c|}
\hline & \multicolumn{6}{|c|}{ SAMPLE ISOTOPIC COMPOSITION } & \multicolumn{2}{|c|}{ INTERNAL STD. } \\
\hline PNNL No. & ${ }^{234} \mathrm{U} /{ }^{235} \mathrm{U}$ & $\underline{1-\sigma \text { Error }}$ & ${ }^{238} \mathrm{U} /{ }^{235} \mathrm{U}$ & $\underline{1-\sigma \text { Error }}$ & ${ }^{236} \mathrm{U} /{ }^{235} \mathrm{U}$ & $1-\sigma$ Error & ${ }^{236} \mathrm{U} /{ }^{233} \mathrm{U}$ & $1-\sigma$ Error \\
\hline 81455 & 0007558 & 0000044 & 13804 & 015 & & & 00517 & 00017 \\
\hline 81456 & 0.007591 & 0.000054 & 138.63 & 0.15 & & & 0.94960 & 0.00078 \\
\hline 81457 & 0.007404 & 0.000068 & 138.12 & 0.15 & & & 0.9432 & 0.0010 \\
\hline 81467 & 0.007666 & 0.000069 & 138.37 & 0.15 & 0.001682 & 0.000041 & & \\
\hline 81468 & 0.007649 & 0.000068 & 138.40 & 0.15 & 0.001703 & 0.000040 & & \\
\hline Report: & 0.007576 & 0.000036 & 138.49 & 0.14 & 0.001693 & 0.000029 & 0.9492 & 0.0017 \\
\hline
\end{tabular}


IAEA/NWAL SAMPLE SET OF 13OCT1998: IAEA/NWAL Sample No. IAEA-2 CORRECTED FOR ISOTOPIC FRACTIONATION USING INTERNAL ${ }^{236} U /{ }^{233} U$ STANDARD IAEA/LLNL

\begin{tabular}{|c|c|c|c|c|c|c|c|c|}
\hline & \multirow{2}{*}{\multicolumn{6}{|c|}{ SAMPLE ISOTOPIC COMPOSITION }} & \multirow{2}{*}{\multicolumn{2}{|c|}{ INTERNAL STD. }} \\
\hline & & & & & & & & \\
\hline PNNL No. & ${ }^{234} \mathrm{U} /{ }^{235} \mathrm{U}$ & $\underline{1-\sigma \text { Error }}$ & ${ }^{238} \mathrm{U} /{ }^{235} \mathrm{U}$ & $1-\sigma$ Error & ${ }^{236} \mathrm{U} /{ }^{235} \mathrm{U}$ & $\underline{1-\sigma \text { Error }}$ & ${ }^{236} \mathrm{U} /{ }^{233} \mathrm{U}$ & $\underline{1-\sigma \text { Error }}$ \\
\hline 81458 & 0.007603 & 0.000070 & 136.63 & 0.23 & & & & \\
\hline 81459 & 0.007677 & 0.000085 & 136.54 & 0.24 & & & & \\
\hline 81460 & 0.007587 & 0.000071 & 132.92 & 0.23 & & & & \\
\hline \multirow{2}{*}{ Report: } & 0.007616 & 0.000043 & 135.33 & 1.22 & & & 0.94967 & 0.00018 \\
\hline & & & & & & & \multicolumn{2}{|c|}{ (Above Value is Given) } \\
\hline
\end{tabular}

\begin{tabular}{|c|c|c|c|c|c|c|c|c|}
\hline \multicolumn{9}{|c|}{ IAEA/NWAL SAMPLE SET OF 130CT1998: IAEA/NWAL Sample No. IAEA-2 } \\
\hline \multicolumn{9}{|c|}{ NOT CORRECTED FOR ISOTOPIC FRACTIONATION } \\
\hline & \multirow{2}{*}{\multicolumn{6}{|c|}{ SAMPLE ISOTOPIC COMPOSITION }} & \multirow{2}{*}{\multicolumn{2}{|c|}{ INTERNAL STD. }} \\
\hline & & & & & & & & \\
\hline PNNL No. & 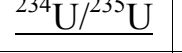 & $\underline{1-\sigma \text { Error }}$ & ${ }^{238} \mathrm{U} /{ }^{230} \mathrm{U}$ & $\underline{1-\sigma \text { Error }}$ & ${ }^{236} \mathrm{U} /{ }^{235} \mathrm{U}$ & $1-\sigma$ Error & ${ }^{236} \mathrm{U} /{ }^{233} \mathrm{U}$ & $\underline{1-\sigma \text { Error }}$ \\
\hline 81458 & 0.007582 & 0.000070 & 135.56 & 0.14 & & & 0.9420 & 0.0011 \\
\hline 81459 & 0.007659 & 0.000072 & 135.79 & 0.15 & & & 0.9442 & 0.0013 \\
\hline 81460 & 0.007506 & 0.000070 & 135.69 & 0.15 & & & 0.9695 & 0.0012 \\
\hline 81469 & 0.007699 & 0.000072 & 135.79 & 0.15 & 0.001864 & 0.000046 & & \\
\hline 81470 & 0.007588 & 0.000075 & 135.52 & 0.15 & 0.001899 & 0.000048 & & \\
\hline Report: & $\mathbf{0 . 0 0 7 5 8 8}$ & 0.000032 & 135.67 & 0.07 & 0.001881 & 0.000033 & 0.9517 & 0.0088 \\
\hline
\end{tabular}




\begin{tabular}{|c|c|c|c|c|c|c|c|c|}
\hline \multirow{2}{*}{\multicolumn{9}{|c|}{\begin{tabular}{|l|} 
IAEA/NWAL SAMPLE SET OF \\
CORRECTED FOR ISOTO \\
STANDARD IAEA/LLNL \\
\end{tabular}}} \\
\hline & & & & & & & & \\
\hline & & & & & & & & \\
\hline \multirow[b]{2}{*}{ PNNL No. } & \multicolumn{6}{|c|}{ SAMPLE ISOTOPIC COMPOSITION } & \multicolumn{2}{|c|}{ INTERNAL STD. } \\
\hline & ${ }^{234} \mathrm{U} /{ }^{235} \mathrm{U}$ & $1-\sigma$ Error & ${ }^{238} \mathrm{U} /{ }^{235} \mathrm{U}$ & $1-\sigma$ Error & ${ }^{236} \mathrm{U} /{ }^{235} \mathrm{U}$ & $1-\sigma$ Error & ${ }^{236} \mathrm{U} /{ }^{233} \mathrm{U}$ & $\underline{1-\sigma \text { Error }}$ \\
\hline 81461 & 0.007730 & 0.000070 & 133.22 & 0.21 & & & & \\
\hline 81462 & 0.007699 & 0.000070 & 132.84 & 0.21 & & & & \\
\hline 81463 & 0.007707 & 0.000071 & 134.15 & 0.22 & & & & \\
\hline Report: & 0.007712 & 0.000041 & 133.38 & 0.39 & & & 0.94967 & 0.00018 \\
\hline & & & & & & & (Above Valt & e is Given) \\
\hline
\end{tabular}

\begin{tabular}{|c|c|c|c|c|c|c|c|c|}
\hline \multicolumn{9}{|c|}{ IAEA/NWAL SAMPLE SET OF 13OCT1998: IAEA/NWAL Sample No. IAEA-3 } \\
\hline \multicolumn{9}{|c|}{ NOT CORRECTED FOR ISOTOPIC FRACTIONATION } \\
\hline & \multirow{2}{*}{\multicolumn{6}{|c|}{ SAMPLE ISOTOPIC COMPOSITION }} & \multirow{2}{*}{\multicolumn{2}{|c|}{ INTERNAL STD. }} \\
\hline & & & & & & & & \\
\hline PNNL No. & ${ }^{234} \mathrm{U} /{ }^{235} \mathrm{U}$ & $\underline{1-\sigma \text { Error }}$ & ${ }^{238} \mathrm{U} /{ }^{235} \mathrm{U}$ & $\underline{1-\sigma \text { Error }}$ & ${ }^{236} \mathrm{U} /{ }^{235} \mathrm{U}$ & $1-\sigma$ Error & ${ }^{236} \mathrm{U} /{ }^{233} \mathrm{U}$ & $\underline{1-\sigma \text { Error }}$ \\
\hline 81461 & 0.007698 & 0.000070 & 132.95 & 0.14 & & & 0.94750 & 0.00098 \\
\hline 81462 & 0.007665 & 0.000070 & 133.23 & 0.14 & & & 0.95221 & 0.00099 \\
\hline 81463 & 0.007700 & 0.000070 & 132.76 & 0.14 & & & 0.93951 & 0.00098 \\
\hline 81471 & 0.007617 & 0.000072 & 133.16 & 0.14 & 0.001932 & 0.000045 & & \\
\hline 81472 & 0.007772 & 0.000069 & 132.95 & 0.14 & 0.002070 & 0.000045 & & \\
\hline Report: & 0.007692 & 0.000031 & 133.01 & 0.08 & 0.002001 & .0000069 & 0.9464 & 0.0037 \\
\hline
\end{tabular}

Average values for spiked samples with spike correction (editor's addition).

$\begin{array}{llll}\text { Sample } & \text { R8/5 } & 2 \text { st dev } \begin{array}{l}\text { corr/ } \\ \text { uncor }\end{array} \\ & & & \\ 1 & 138.68 & 0.77 & 1.0008 \\ 2 & 136.59 & 0.13 & 1.0067 \text { one value excluded } \\ 3 & 133.40 & 1.35 & 1.0032\end{array}$




\section{Appendix E}

\section{Reports from Laboratory $L$}

(Reformatted to fit pages)

DOUBLE SPIKE RESULTS

Name Deleted

October, 1998

\begin{tabular}{|c|c|c|c|c|c|}
\hline \multirow{2}{*}{ Sample } & \multicolumn{3}{|c|}{ LLNL Spike } & \multicolumn{2}{|c|}{ IAEA Spike } \\
\hline & Filament & $235 / 238$ & $\mathrm{CR}{ }^{238} \mathrm{U}^{+}$ & $235 / 238$ & $\mathrm{CR}^{238} \mathrm{U}^{+}$ \\
\hline \multirow[t]{6}{*}{1} & 1 & 0.007155 & 1E5 & 0.007156 & $3 E 5$ \\
\hline & 2 & 0.007150 & 4E4 & 0.007146 & 4E5 \\
\hline & 3 & 0.007191 & 4E4 & 0.007114 & 2E5 \\
\hline & 4 & 0.007177 & $5 \mathrm{E} 4$ & & \\
\hline & Avg & 0.007168 & & 0.007139 & \\
\hline & SD & 0.000019 & & 0.000022 & \\
\hline \multirow[t]{6}{*}{2} & 1 & 0.007347 & 7E4 & 0.007324 & 3E5 \\
\hline & 2 & 0.007310 & 4E4 & 0.007286 & $2 \mathrm{E} 5$ \\
\hline & 3 & 0.007346 & 4E4 & 0.007293 & $2 \mathrm{E} 5$ \\
\hline & 4 & 0.007325 & 4E4 & & \\
\hline & Avg & 0.007332 & & 0.007301 & \\
\hline & SD & 0.000018 & & 0.000020 & \\
\hline \multirow[t]{5}{*}{3} & 1 & 0.007466 & $8 \mathrm{E} 4$ & 0.007522 & $2 \mathrm{E} 5$ \\
\hline & 2 & 0.007470 & 5E4 & 0.007497 & 2E5 \\
\hline & 3 & 0.007506 & 8E4 & 0.007494 & 2E5 \\
\hline & Avg & 0.007481 & & 0.007505 & \\
\hline & SD & 0.000022 & & 0.000015 & \\
\hline
\end{tabular}

UNSPIKED RESULTS

$\begin{array}{rlllll}\text { Sample } & \underline{234} & \underline{235} & \underline{236} & \underline{238} & \underline{235 / 238} \\ 1 & 0.0054 & 0.7080 & 0.0012 & 99.2854 & 0.007131 \\ 2 & 0.0055 & 0.7205 & 0.0015 & 99.2725 & 0.007258 \\ 3 & 0.0057 & 0.7259 & 0.0015 & 99.2670 & 0.007313\end{array}$


RESULTS: DOUBLE SPIKE EXPERIMENT

$\begin{array}{lllll}\text { Sample } & 5 / 8 \mathrm{UL} & 5 / 8 \mathrm{UI} & 5 / 8 \mathrm{CL} & 5 / 8 \mathrm{Cl} \\ \text { 1A } & 0.00718 & 0.00713 & 0.007155 & 0.007156 \\ \text { 1B } & 0.00729 & 0.00715 & 0.007150 & 0.007146 \\ \text { 1C } & 0.00719 & 0.00714 & 0.007191 & 0.007114 \\ \text { 1D } & 0.00719 & 0.00703 & 0.007177 & \\ \text { Avg } & 0.00722 & 0.00710 & 0.007168 & 0.007139 \\ \text { SD } & 0.00005 & 0.00006 & 0.000019 & 0.000022\end{array}$

$\begin{array}{lllll}\text { 2A } & 0.00733 & 0.00736 & 0.007347 & 0.007324 \\ \text { 2B } & 0.00731 & 0.00727 & 0.007310 & 0.007286 \\ \text { 2C } & 0.00736 & 0.00725 & 0.007346 & 0.007293 \\ \text { 2D } & 0.00731 & 0.00735 & 0.007325 & \\ \text { Avg } & 0.00733 & 0.00731 & 0.007332 & 0.007301 \\ \text { SD } & 0.00002 & 0.00005 & 0.000018 & 0.000020\end{array}$

$\begin{array}{lllll}\text { 3A } & 0.00750 & 0.00745 & 0.007466 & 0.007522 \\ \text { 3B } & 0.00753 & 0.00746 & 0.007470 & 0.007497 \\ \text { 3C } & 0.00750 & 0.00746 & 0.007506 & 0.007494 \\ \text { Avg } & 0.00751 & 0.00746 & 0.007481 & 0.007505 \\ \text { SD } & 0.00002 & 0.00001 & 0.000022 & 0.000015\end{array}$

Notes: UL are results using the LLNL spike but without internal calibration applied. $\mathrm{UI}$ are results using the IAEA spike but without internal calibraion applied. $\mathrm{CL}$ are results using the LLNL spike with internal calibration applied. $\mathrm{Cl}$ are results using the IAEA spike with internal calibration applied. 


\section{MORE DOUBLE SPIKE RESULTS}

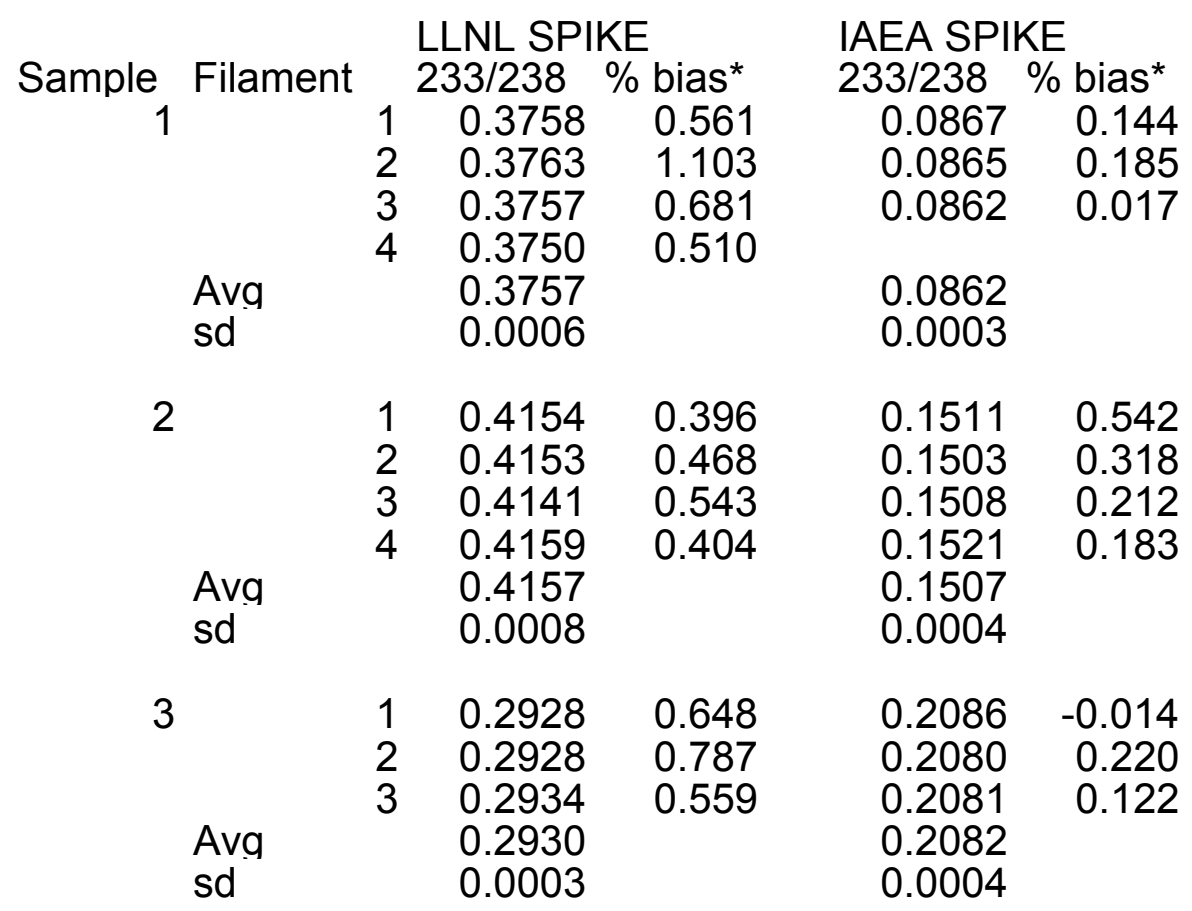

\section{* per mass unit}

Some observations:

1) It is clear that the heavier sample loading with the IAEA spike resulted in lower average bias corrections. I assume it was in part because temperatures were lower. This clear-cut difference is a good illustration of the power of the double spike: You don't need to run at the same temperature and count rate to get good results.

2) I imagine our bias will be higher than most labs' because we scan the high voltage rather than the magnet. This is to maximize the amount of data we can collect on small samples. You can't (sigh) have everything.

3) I assume you know this, but I'll spell it out to be sure there is no misunderstanding. To get the bias applied to the $235 / 238$ ratio using the bias correction per mass, the following tough equation is used: $\operatorname{bias}(5 / 8)=1.000-3 *$ fractional bias correction per mass. This bias is then multiplied by the raw counts of 235 before the ratio is calculated.

4) As always, I found the range of the bias corrections rather shocking: $-0.014 \%$ to $1.103 \%$. No wonder we have trouble sometimes!

5) As I mentioned on the phone, ideal samples like these do not present the best test case for the double spike. A double spike would hardly be needed if all samples were as clean as these. Maybe a follow-up experiment using a gloppy sample(s) is in order--assuming all DOE labs agree well. 


\section{DOUBLE SPIKE RESULTS (REPEAT)}

Note: IAEA spike was used.

\begin{tabular}{|c|c|c|c|}
\hline Sample & Filament & $235 / 238$ & $\mathrm{CR},{ }^{238} \mathrm{~L}$ \\
\hline \multirow[t]{6}{*}{1} & 1 & $\overline{0.007120}$ & $4 \mathrm{E} 5$ \\
\hline & 2 & 0.007147 & $5 E 5$ \\
\hline & 3 & 0.007144 & $2 \mathrm{E} 5$ \\
\hline & Avg & 0.007137 & \\
\hline & $S D$ & 0.000015 & \\
\hline & RSD & $0.2 \%$ & \\
\hline \multirow[t]{6}{*}{2} & 1 & 0.007298 & 5E5 \\
\hline & 2 & 0.007285 & 4E5 \\
\hline & 3 & 0.007295 & $1 \mathrm{E} 5$ \\
\hline & Avg & 0.007293 & \\
\hline & SD & 0.000007 & \\
\hline & RSD & $0.09 \%$ & \\
\hline \multirow[t]{6}{*}{3} & 1 & 0.007439 & $2 \mathrm{E} 5$ \\
\hline & 2 & 0.007427 & $6 E 5$ \\
\hline & 3 & 0.007436 & $1 \mathrm{E} 5$ \\
\hline & Avg & 0.007434 & \\
\hline & SD & 0.000006 & \\
\hline & RSD & $0.08 \%$ & \\
\hline
\end{tabular}

